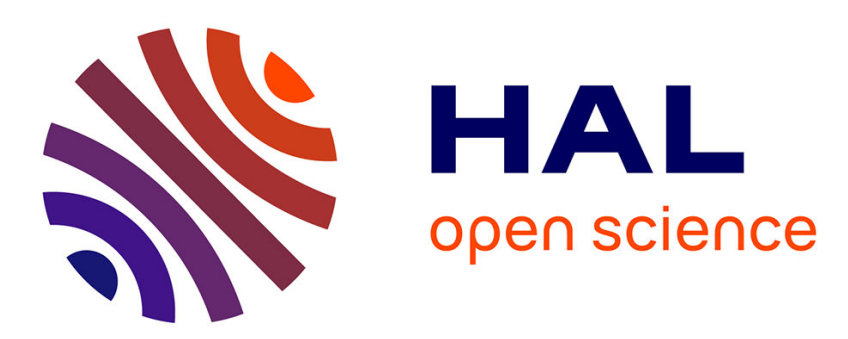

\title{
Sequential aza-Baylis-Hillman/Ring closing metathesis/aromatization as a novel route for the synthesis of substituted pyrroles
}

Valérie Declerck, Patrice Ribière, Jean Martinez, Frédéric Lamaty

\section{To cite this version:}

Valérie Declerck, Patrice Ribière, Jean Martinez, Frédéric Lamaty. Sequential aza-BaylisHillman/Ring closing metathesis/aromatization as a novel route for the synthesis of substituted pyrroles. Journal of Organic Chemistry, 2004, 69 (24), pp.8372-8381. 10.1021/jo048519r . hal00077977

\section{HAL Id: hal-00077977 \\ https://hal.science/hal-00077977}

Submitted on 5 Feb 2021

HAL is a multi-disciplinary open access archive for the deposit and dissemination of scientific research documents, whether they are published or not. The documents may come from teaching and research institutions in France or abroad, or from public or private research centers.
L'archive ouverte pluridisciplinaire HAL, est destinée au dépôt et à la diffusion de documents scientifiques de niveau recherche, publiés ou non, émanant des établissements d'enseignement et de recherche français ou étrangers, des laboratoires publics ou privés. 


\title{
Sequential aza-Baylis-Hillman/Ring Closing Metathesis/ Aromatization as a Novel Route for the Synthesis of Substituted Pyrroles
}

\author{
Valérie Declerck, Patrice Ribière, Jean Martinez, and Frédéric Lamaty* \\ Laboratoire des Aminoacides, Peptides et Protéines (LAPP), CNRS-Universités Montpellier 1 et 2 , \\ Place Eugène Bataillon, 34095 Montpellier Cedex 5, France
}

frederic@univ-montp2.fr

\begin{abstract}
A new route to diverse 2-substituted-3-methoxycarbonyl pyrroles has been developed. Diverse SES protected $\alpha$-methylene $\beta$-aminoesters were obtained by a 3 -component $a z \alpha$-Baylis-Hillman reaction. Diversity arose from the aryl aldehydes which can be used in this reaction. $N$-Alkylation with allyl bromide under mild conditions provided the corresponding dienes. These substituted dienes were cyclized by ring closing metathesis at room temperature or under microwave-activation with Grubbstype II catalyst to yield SES-protected pyrroline intermediates. The final pyrroles were obtained by base-promoted dehydrodesulfinylation/aromatization. The scope of each of these reactions was explored.
\end{abstract}

\section{Introduction}

The 2-trimethylsilyethylsulfonyl (or SES) group is a valuable protecting group of amines in organic synthesis. ${ }^{1}$ It is mainly used to protect an amine as a sulfonamide and promote Mitsunobu or base-activated alkylation. This group can be cleaved usually by fluoride-promoted $\beta$-elimination. A less usual pathway to remove the SES group is to perform a base-promoted dehydrodesulfinylation, which consists of abstracting a proton in the $\alpha$-position to the nitrogen of the sulfonamide. This process is favored in the case of unsaturated cyclic compounds when an aromatization to the final product is possible. It has been described in the literature mostly with the tosyl $^{2}$ or related protecting groups ${ }^{2 \mathrm{~d}, 3}$ and more rarely with the SES group ${ }^{3 \mathrm{k}, 4}$ (eq 1).

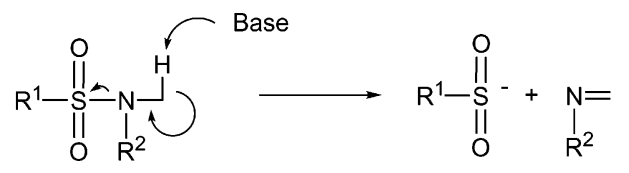

As part of our ongoing project on the synthesis of heterocyclic structures by ring closing metathesis $(\mathrm{RCM}),{ }^{4 \mathrm{~b}, 5}$ we report herein a new strategy for the preparation of 2,3-disubstituted pyrroles using the dehydrodesulfinylation as the aromatization step of pyrrolines formed by RCM. The pyrrole ${ }^{6}$ ring is an important scaffold in pharmaceutical and material chemistry. It is

* Address correspondence to this author. Fax: +33 (0) 4-67-14-48-

(1) (a) Weinreb, S. M.; Ralbovsky, J. L. In Handbook of Reagents for Organic Synthesis, Activating Agents and Protecting Groups; Pearson, A. J., Roush, W. J., Eds.; Wiley: Chichester, UK, 1999; pp 425-427. (b) In Protective Groups in Organic Synthesis, 3rd ed.; Greene, T. W., Wuts, P. G. M., Eds.; Wiley: New York, 1999; p 612. (c) Kocienski, P. J. In Protecting Groups; Enders, D., Noyori, R., Trost, B. M., Eds.; Thieme: New York, 2000; pp 215-216. found in biomolecules ${ }^{6 a, b}$ such as porphyrins and cytochromes. It serves also as a building block for the preparation of polymeric and supramolecular structures which have applications in nonlinear optics. ${ }^{6 c, d}$

\section{Results and Discussion}

As described in the retrosynthetic scheme (Scheme 1), the pyrrole ring can be obtained after dehydrodesulfin-

(2) (a) Holmes, E. L.; Ingold, C. K. J. Chem. Soc. 1926, 1305-1310. (b) Birch, A. J.; Jackson, A. H.; Shannon, P. V. R. J. Chem. Soc., Perkin Trans. 1 1974, 2185-2190. (c) Birch, A. J.; Jackson, A. H.; Shannon P. V. R. J. Chem. Soc., Perkin Trans. 1 1974, 2190-2194. (d) Rozwadowska, M. D.; Brozda, D. Can. J. Chem. 1980, 58, 1239-1242. (e) Boger, D. L; Brotherton, C. E. Kelley, M. D. Tetrahedron 1981, 37, 3977-3980. (f) McKay, W. R.; Proctor, G. R. J. Chem. Soc., Perkin Trans. 1 1981, 2435-2442. (g) McKay, W. R.; Proctor, G. R. J. Chem. Soc., Perkin Trans. 1 1981, 2443-2450. (h) Veeraraghavan, S.; Popp, F. D. J. Heterocycl. Chem. 1981, 18, 775-777. (i) Bradamante, S.; Colombo, S.; Pagani, G. A.; Roelens, S. Helv. Chim. Acta 1981, 64, 2524-2527. (j) Blaikley, D. C. W.; Currie, D. W.; Smith, D. M.; Watson, S. A.; McNab, H. J. Chem. Soc., Perkin Trans. 1 1984, 367-369. (k) Boger, D. L.; Brotherton, C. E.; Panek, J. S.; Yohannes, D. J. Org. Chem. 1984, 49, 4056-4058. (1) Harrison, D. M.; Sharma, R. B. Tetrahedron Lett. 1986, 27, 521-524. (m) Hogan, I.; Jenkins, P.; Sainsbury, M. Tetrahedron Lett. 1988, 29, 6505-6508. (n) Hogan, I. Jenkins, P. D.; Sainsbury, M. Tetrahedron 1990, 46, 2943-2964. (o) Harrison, D. M.; Sharma, R. B. Tetrahedron 1993, 49, 3165-3184. (p) Boogaard, A. T.; Pandit, U. K.; Koomen, G. J. Tetrahedron 1994, 50, 2551-2560. (q) Garcia, A.; Castedo, L.; Dominguez, D. Tetrahedron 1995, 51, 8585-8598. (r) Davis, F. A.; Liang, C.-H.; Liu, H. J. Org. Chem. 1997, 62, 3796-3797. (s) Meng, Q.; Thibblin, A. J. Am. Chem. Soc. 1997, 119, 1224-1229. (t) Dube, D.; Blouin, M.; Brideau, C.; Chan, C.-C.; Desmarais, S.; Ethier, D.; Falgueyret, J.-P.; Friesen, R. W.; Girard, M.; Girard, Y.; Guay, J.; Riendeau, D.; Tagari, P.; Young, R. N. Bioorg. Med. Chem. Lett. 1998, 8, 1255-1260. (u) Watson, T. J. N. J. Org. Chem. 1998, 63, 406-407. (v) Davis, F. A.; Liu, H.; Liang, C.H.; Reddy, G. V.; Zhang, Y.; Fang, T.; Titus, D. D. J. Org. Chem. 1999, 64, 8929-8935. (w) Nandi, B.; Kundu, N. G. Org. Lett. 2000, 2, 235238. (x) Kim, J. N.; Lee, H. J.; Lee, K. Y.; Kim, H. S. Tetrahedron Lett. 2001, 42, 3737-3740. (y) Kundu, N. G.; Nandi, B. J. Org. Chem. 2001, 66, 4563-4575. (z) Lee, J. C.; Cha, J. K. J. Am. Chem. Soc. 2001, 123 3243-3246. (aa) Tokuyama, H.; Sato, M.; Ueda, T.; Fukuyama, T. Heterocycles 2001, 54, 105-108. (ab) Silveira, C. C.; Bernardi, C. R. Braga, A. L.; Kaufman, T. S. Tetrahedron Lett. 2001, 42, 8947-8950. (ac) Silveira, C. C.; Bernardi, C. R.; Braga, A. L.; Kaufman, T. S. Synlett 2002, 907-910. 


\section{SCHEME 1}

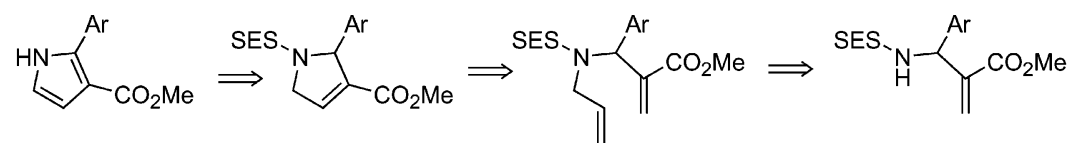

ylation/aromatization of a SES-protected pyrroline formed via RCM of an appropriate linear precursor. This precursor can result from the alkylation of an unsaturated $\beta$-aminoester synthesized by the $a z a$ Baylis-Hillman reaction. Recently we have presented the synthesis of an ester-substituted pyrroline via RCM. ${ }^{5 b}$ To increase the diversity of this class of molecules ${ }^{7}$ as well as the pyrrole derivatives obtained by aromatization, we needed to prepare more diverse $\alpha$-methylene- $\beta$ aminoesters which can be obtained via the $a z a$-BaylisHillman reaction.

The 3-component aza version of the Baylis-Hillman reaction is an attractive method for the synthesis of $\beta$-aminoesters. This reaction has been performed with the tosyl group $\left(\mathrm{R}^{3}=\right.$ tosyl) as the protecting and activating group of ammonia. ${ }^{8}$ With the purpose of synthesizing $\beta$-aminoesters bearing more easily cleavable protecting groups, we decided to investigate the SES group for protection (eq 2). ${ }^{9}$

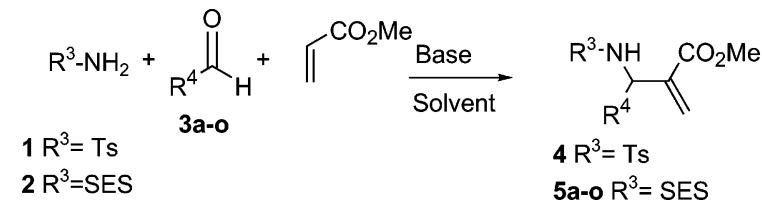

First, the conditions by Balan et al. ${ }^{8 b}$ for $\mathrm{Ts}_{\mathrm{s}} \mathrm{NH}_{2}$ were tested with use of SES-NH acrylate in the presence of a Lewis acid $\left(\mathrm{Ti}\left(\mathrm{O} i-\mathrm{Pr}_{4}\right)\right)$,

(3) (a) Mertes, M. P.; Borne, R. F.; Hare, L. E. J. Org. Chem. 1968 , 33, 133-137. (b) Hendrickson, J. B.; Bergeron, R.; Giga, A.; Sternbach, D. J. Am. Chem. Soc. 1973, 95, 3412-3413. (c) Aratani, M.; Hashimoto, M. J. Am. Chem. Soc. 1980, 102, 6171-6172. (d) Kreher, R.; Gerhardt, W. Liebigs Ann. Chem. 1981, 240-247. (e) Boger, D. L.; Zhang, M. J. Org. Chem. 1992, 57, 3974-3977. (f) Boger, D. L.; Corbett, W. L. J. Org. Chem. 1993, 58, 2068-2074. (g) Kohno, H.; Yamada, K. Heterocycles 1999, 51, 103-117. (h) Haase, M.; Gunther, W.; Gorls, H. Anders, E. Synthesis 1999, 2071-2081. (i) Engler, T. A.; Wanner, J. J. Org. Chem. 2000, 65, 2444-2457. (j) Katritzky, A. R.; Zhang, S.; Kurz, T.; Wang, M.; Steel, P. J. Org. Lett. 2001, 3, 2807-2809. (k) Parker, K. A.; Mindt, T. L. Org. Lett. 2002, 4, 4265-4268. (1) Marcotte, F.-A.; Lubell, W. D. Org. Lett. 2002, 4, 2601-2603. (m) Marcotte, F.A.; Rombouts, F. J. R.; Lubell, W. D. J. Org. Chem. 2003, 68, 69846987. (n) Jeannotte, G.; Lubell, W. D. J. Org. Chem. 2004, 69, 46564662.

(4) (a) Xu, Z.; Lu, X. J. Org. Chem. 1998, 63, 5031-5041. (b) Varray, S.; Lazaro, R.; Martinez, J.; Lamaty, F. Eur. J. Org. Chem. 2002, 23082316.

(5) (a) Varray, S.; Gauzy, C.; Lamaty, F.; Lazaro, R.; Martinez, J. J. Org. Chem. 2000, 65, 6787-6790. (b) Varray, S.; Lazaro, R.; Martinez, J.; Lamaty, F. Organometallics 2003, 22, 2426-2435.

(6) For most recent work, see: (a) Tracey, M. R.; Hsung, R. P. Lambeth, R. H. Synthesis 2004, 918-922. (b) Dhawan, R.; Arndtsen, B. A. J. Am. Chem. Soc. 2004, 126, 468-469 (c) Venkatraman, S.; Kumar, R.; Sankar, J.; Chandrashekar, T. K.; Sendhil, K.; Vijayan, C.; Kelling, A.; Senge, M. O. Chem. Eur. J. 2004, 10, 1423-1432. (d) Facchetti, A.; Abbotto, A.; Beverina, L.; van der Boom, M. E.; Dutta, P.; Evmenenko, G.; Pagani, G. A.; Marks, T. J. Chem. Mater. 2003 15, 1064-1072 and references therein.

(7) During the course of our study, a similar approach for the synthesis of pyrrolines was published: Balan, D.; Adolfsson, H. Tetrahedron Lett. 2004, 45, 3089-3092.

(8) (a) Balan, D.; Adolfsson, H. J. Org. Chem. 2001, 66, 6498-501. (b) Balan, D.; Adolfsson, H. J. Org. Chem. 2002, 67, 2329-34. (c) Balan, D.; Adolfsson, H. Tetrahedron Lett. 2003, 44, 2521-2524.

(9) For a related approach on PEG-support: Ribière, P.; Enjalbal, C.; Aubagnac, J.-L.; Yadav-Bhatnagar, N.; Martinez, J.; Lamaty, F. J. Comb. Chem. 2004, 6, 464-467.
TABLE 1.

\begin{tabular}{|c|c|c|c|c|c|c|c|}
\hline \multirow[b]{3}{*}{$\mathrm{R}^{4}$} & \multicolumn{4}{|c|}{ yield $a$ of $5(\%)$} & \multirow[b]{3}{*}{$\begin{array}{c}\text { yield of } \\
6(\%)\end{array}$} & \multirow[b]{3}{*}{$\begin{array}{c}\text { yield }^{a} \text { of } \\
7(\%)\end{array}$} & \multirow[b]{3}{*}{$\begin{array}{c}\text { yield }^{a} \text { of } \\
\mathbf{8}(\%)\end{array}$} \\
\hline & \multicolumn{2}{|c|}{ conditions $\mathrm{A}^{g}$} & \multicolumn{2}{|c|}{ conditions $\mathrm{B}^{g}$} & & & \\
\hline & $t(\mathrm{~h})$ & $\begin{array}{c}\text { yield } \\
(\%)\end{array}$ & $t(\mathrm{~h})$ & $\begin{array}{c}\text { yield } \\
(\%)\end{array}$ & & & \\
\hline $\mathbf{a}$ & 24 & 73 & 10 & 86 & 98 & 98 & 80 \\
\hline b & 96 & 46 & 72 & 67 & 98 & 95 & 83 \\
\hline $\mathbf{c}$ & 48 & 64 & 6 & 90 & 97 & 92 & $b$ \\
\hline d & 24 & 75 & \multicolumn{2}{|r|}{$c$} & $c$ & $c$ & $c$ \\
\hline $\mathbf{e}$ & 72 & 36 & 6 & 79 & 99 & 95 & 81 \\
\hline $\mathbf{f}$ & 72 & 42 & 6 & 86 & 99 & 90 & 81 \\
\hline $\mathbf{g}$ & \multicolumn{2}{|r|}{$d$} & 48 & 60 & 98 & 90 & 66 \\
\hline $\mathbf{h}$ & \multicolumn{2}{|r|}{$c$} & 38 & 86 & 98 & 98 & 72 \\
\hline i & \multicolumn{2}{|r|}{$c$} & 72 & 70 & 97 & 92 & 78 \\
\hline $\mathbf{j}$ & \multicolumn{2}{|r|}{$c$} & 72 & 66 & 98 & 96 & 88 \\
\hline $\mathbf{k}$ & \multicolumn{2}{|r|}{$d$} & 14 & 86 & 99 & 95 & 81 \\
\hline 1 & \multicolumn{2}{|r|}{$d$} & 10 & 73 & 99 & 94 & 81 \\
\hline $\mathbf{m}$ & \multicolumn{2}{|r|}{$d$} & 14 & 61 & 98 & $5^{f}$ & \\
\hline $\mathbf{n}$ & \multicolumn{2}{|r|}{$d$} & 6 & 60 & 97 & $10^{f}$ & \\
\hline $\mathbf{0}$ & \multicolumn{2}{|r|}{$d$} & 6 & 71 & $e$ & & \\
\hline
\end{tabular}

${ }^{a}$ After purification by column chromatography. ${ }^{b}$ Aromatization occurred along with transesterification of the side chain with $t$-BuOH. ${ }^{c}$ Not performed. ${ }^{d}$ Slow reaction at room temperature and degradation of the aldehyde upon heating. ${ }^{e}$ Degradation observed. ${ }^{f}$ Conversion evaluated by ${ }^{1} \mathrm{H}$ NMR. ${ }^{g}$ Conditions A: $\mathrm{SESNH}_{2}(1$ equiv), $\mathrm{ArCHO}$ ( 1 equiv), methyl acrylate (1.1 equiv), 3 -HQD ( 0.15 equiv), $\mathrm{Ti}-(\mathrm{O} i \mathrm{Pr})_{4}\left(0.02\right.$ equiv), $i$-PrOH, MS $4 \AA$, $70^{\circ} \mathrm{C}$. Conditions B: $\mathrm{SESNH}_{2}$ ( 1 equiv), ArCHO (5 equiv), methyl acrylate (5 equiv), $\mathrm{DABCO}$ ( 0.5 equiv), $i-\mathrm{PrOH}, 70{ }^{\circ} \mathrm{C}$.

3-HQD as a base, and molecular sieves. When the reaction was performed at room temperature conversion was slow and selectivity between formation of the $\beta$-aminoester and the $\beta$-hydroxyester was moderate. Increasing the reaction temperature to $70{ }^{\circ} \mathrm{C}$ resulted in complete conversion after $24 \mathrm{~h}$ and better selectivity. Reaction of SES- $\mathrm{NH}_{2}$ was slower than that with $\mathrm{Ts}-\mathrm{NH}_{2}$ ( $8 \mathrm{~h}$ in the same reaction conditions). We applied these reaction conditions to the synthesis of six aminoesters $(\mathbf{5 a}-\mathbf{f})$. However, we realized that in some cases, the reaction time and conditions were not satisfactory. With some aldehydes, the reaction was too slow and incomplete after $24 \mathrm{~h}$. Heating the reaction mixture resulted in the degradation of the reactants. Consequently we also performed the reactions in different conditions (DABCO in $i$-PrOH) in the presence of an excess of benzaldehyde and methyl acrylate to drive the reaction to completion. Concomitant formation of the corresponding hydroxyester was not avoided. The aminoesters were obtained pure after column chromatography. Results are presented in Table 1.

The various $\beta$-aminoesters obtained from the $a z a$ Baylis-Hillman reaction $(\mathbf{5 a}-\mathbf{n})$ were reacted with allyl bromide in the presence of $\mathrm{K}_{2} \mathrm{CO}_{3}$ in $\mathrm{DMF}^{5 \mathrm{~b}}$ to yield the corresponding dienes in most cases, except for $\mathbf{5 d}$, which yielded a complex mixture, and 5o, which was degraded in these reaction conditions. $6 \mathbf{a}-\mathbf{c}$ and $\mathbf{6 e}-\mathbf{n}$ were obtained in almost quantitative yields.

Ring closing metathesis is a powerful method for the construction of cyclic structures. ${ }^{10}$ In the past few years, it has been widely applied to the synthesis of hetero- 


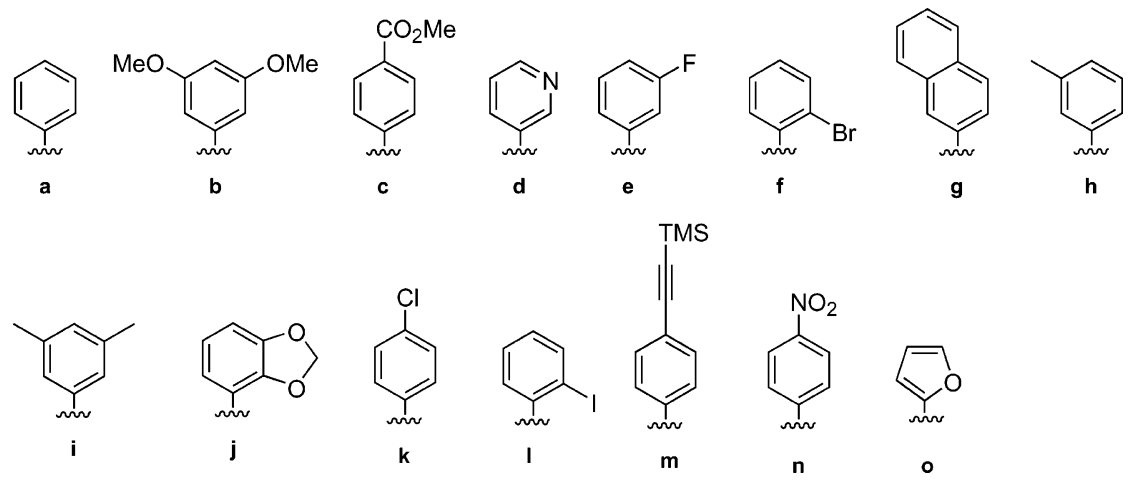

FIGURE 1. $R^{4}$ substituents.

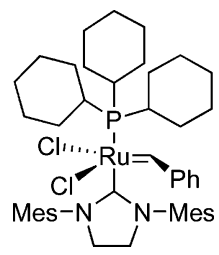

TABLE 2. Yield ${ }^{a}$ of Products Obtained at the SES Cleavage/Aromatization Step of $7 a$

\begin{tabular}{|c|c|c|c|c|}
\hline \multirow[b]{2}{*}{ entry } & \multirow[b]{2}{*}{ conditions } & \multicolumn{3}{|c|}{ yield $(\%)$} \\
\hline & & $7 \mathbf{a}$ & pyrrole $\mathbf{8 a}$ & isomer 9 \\
\hline 1 & $n-\mathrm{Bu}_{4} \mathrm{NF}, \mathrm{THF}, \mathrm{rt}$ & 0 & 21 & 0 \\
\hline 2 & $\mathrm{CsF}, \mathrm{DMF}, 80^{\circ} \mathrm{C}, 2 \mathrm{~h}$ & 0 & 41 & 0 \\
\hline 3 & DBU, THF, rt, $24 \mathrm{~h}$ & 0 & 0 & 26 \\
\hline 4 & $\mathrm{~K}_{2} \mathrm{CO}_{3}, \mathrm{DMF}, 80^{\circ} \mathrm{C}, 24 \mathrm{~h}$ & 0 & 0 & 24 \\
\hline 5 & DBU, THF, reflux, 16 h & 0 & 64 & 0 \\
\hline 6 & $t$-BuOK, DMF, rt, $2 \mathrm{~h}$ & 0 & 83 & 0 \\
\hline
\end{tabular}

${ }^{a}$ Yields were determined by ${ }^{1} \mathrm{H}$ NMR with $\mathrm{CH}_{2} \mathrm{Br}_{2}$ as an internal standard.

lysts such as Grubbs-type II catalyst (Figure 2) has opened the path to more demanding processes such as substituent bearing olefins. Among these substituents, carboxymethyl has not been fully exploited so far ${ }^{4 b, 5 b, 12}$ while its importance as a functional group is widely recognized.

The dienes $\mathbf{6 a}-\mathbf{c}$ and $\mathbf{6 e}-\mathbf{n}$ were submitted to RCM reaction conditions (Grubbs-type II catalyst, $5 \mathrm{~mol} \%$ in $\mathrm{CH}_{2} \mathrm{Cl}_{2}$ ) to yield the corresponding cyclic structures 7 (eq 3). Yields are generally excellent (Table 1) except in the

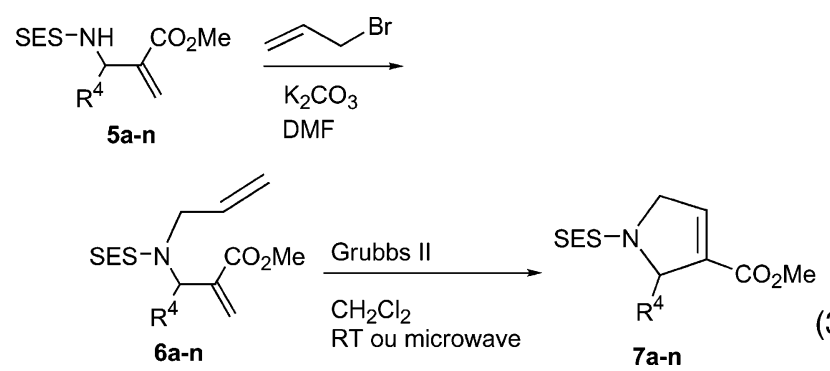

cases of $6 \mathrm{~m}$ and $6 \mathrm{n}$ which gave a low conversion to $7 \mathbf{m}$ and $\mathbf{7 n}$, respectively. This may result from a complexation of the arylic side chain (alkyne ${ }^{13}$ or nitro group)

(10) (a) Schuster, M.; Blechert, S. Angew. Chem., Int. Ed. Engl. 1997, 36, 2036-2055. (b) Ivin, K. J. J. Mol. Catal. A: Chem. 1998, 133, 1-16. (c) Randall, M. L.; Snapper, M. L. J. Mol. Catal. A: Chem 1998, 133 29-40. (d) Armstrong, S. K. J. Chem. Soc., Perkin Trans. 1 1998, 371388. (e) Grubbs, R. H.; Chang, S. Tetrahedron 1998, 54, 4413-4450. (f) Fürstner, A. Top. Organomet. Chem. 1998, 1, 1-231. (g) Trnka, T. N.; Grubbs, R. H. Acc. Chem. Res. 2001, 34, 18-29. (h) Fürstner A. Angew. Chem., Int. Ed. 2000, 39, 3012-3043. (i) Handbook of Metathesis; Grubbs, R. H., Ed.; Wiley-VCH: Weinheim, Germany, 2003; Vol. 2.

(11) (a) Phillips, A. J.; Abell, A. D. Aldrichim. Acta 1999, 32, 7590. (b) Felpin, F.-X.; Lebreton, J. Eur. J. Org. Chem. 2003, 3693-3712. (c) Deiters, A.; Martin, S. F. Chem. Rev. 2004, 104, 2199-2238

(12) (a) Kirkland, T. A.; Grubbs, R. H. J. Org. Chem. 1997, 62, 73107318. (b) Hyldtoft, L.; Madsen, R. J. Am. Chem. Soc. 2000, 122, 84448452. (c) Gessler, S.; Randl, S.; Blechert, S. Tetrahedron Lett. 2000, 41, 9973-9976. (d) Yang, C.; Murray, W. V.; Wilson, L. J. Tetrahedron Lett. 2003, 44, 1783-1786.

with the ruthenium catalyst, unfavorable for the cyclization reaction. While the cylizations of $6 \mathbf{a}-\mathbf{c}$ and $6 \mathbf{e}-\boldsymbol{l}$ were complete within $12 \mathrm{~h}$ at room temperature, they could be conveniently accelerated by microwave activation. ${ }^{5 \mathrm{a}, 7,12 \mathrm{~d}, 14}$ Under these conditions $\left(100{ }^{\circ} \mathrm{C}, \mathrm{CH}_{2} \mathrm{Cl}_{2}\right)$, completion of the cyclization was reached within $5 \mathrm{~min}$.

The next step was the cleavage of the SES group and aromatization (eq 4). Results of the different reaction conditions are summarized in Table 2.

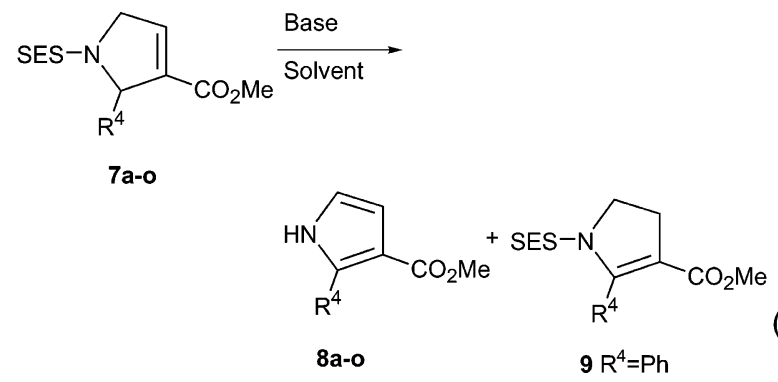

The method described in the literature for this type of substrate $^{4 a}$ (eq 4) yielded only a small amount of the expected product 8a (entry 1). Using a different fluoride source $^{4 b}$ (entry 2 ) resulted in a moderate yield. Stronger bases were then employed. DBU in THF at room temperature ${ }^{2 \mathrm{x}}$ (entry 3 ) and $\mathrm{K}_{2} \mathrm{CO}_{3}$ in DMF at $80{ }^{\circ} \mathrm{C}^{3 \mathrm{k}}$ (entry 4) resulted in the isomerization of pyrroline $\mathbf{7 a}$

(13) Ono, K.; Nagata, T.; Nishida, A. Synlett 2003, 1207-1209.

(14) (a) Mayo, K. G.; Nearhoof, E. H.; Kiddle, J. J. Org. Lett. 2002 4, 1567-1570. (b) Thanh, G. V.; Loupy, A. Tetrahedron Lett. 2003, 44, 9091-9094. (c) Garbacia, S.; Desai, B.; Lavastre, O.; Kappe, C. O. J Org. Chem. 2003, 68, 9136-9139. (d) Efskind, J.; Undheim, K. Tetrahedron Lett. 2003, 44, 2837-2839. (e) Grigg, R.; Martin, W.; Morris, J.; Sridharan, V. Tetrahedron Lett. 2003, 44, 4899-4901. (f) Miles, S. M.; Leatherbarrow, R. J.; Marsden, S. P.; Coates, W. J. Org. Biomol. Chem. 2004, 2, 281-283. (g) Salim, S. S.; Bellingham, R. K.; Brown, R. C. D. Eur. J. Org. Chem. 2004, 4, 800-806. 
into 9 without cleavage of the SES group and aromatization. DBU in refluxing $\mathrm{THF}^{3 \mathrm{e}}$ (entry 5 ) provided better yields of 8a. Best results were obtained with $t$-BuOK in DMF at room temperature ${ }^{2 \mathrm{w}, \mathrm{y}}$ (entry 6). In this case, $83 \%$ of the expected pyrrole $8 \mathbf{a}$ was obtained within $2 \mathrm{~h}$ via elimination/aromatization. This result could be applied to all the SES protected pyrroline $\mathbf{7 a}, \mathbf{b}$ and $\mathbf{7 e}-\boldsymbol{l}$ to provide the corresponding pyrroles $\mathbf{8 a}, \mathbf{b}$ and $\mathbf{8 e}-\boldsymbol{l}$ in high yields. In the case of 7c transesterification of the methyl ester of the side chain to the $t$-Bu ester occurred.

\section{Conclusion}

In conclusion, an efficient regiocontrolled synthesis of 2-substituted-3-methoxycarbonyl pyrroles was developed. Ten different pyrroles were obtained in an overall high yield and high purity, starting from highly diverse $\alpha$-methylene $\beta$-aminoesters. Further development of this chemistry is underway in our laboratory.

\section{Experimental Section}

General Remarks. ${ }^{9}$ Microwave-assisted reactions were performed with a Personal Chemistry Emrys Optimizer or with a CEM Explorer monomode system.

2-(Trimethylsilyl)ethanesulfonamide (2). Phosphorus pentachloride $(7.7 \mathrm{~g}, 36.7 \mathrm{mmol})$ was added portionwise to a suspension of sodium 2-(trimethylsilyl)ethanesulfonate ( $5 \mathrm{~g}$, $24.5 \mathrm{mmol}$ ) in $100 \mathrm{~mL}$ of $\mathrm{CH}_{2} \mathrm{Cl}_{2}$. The reaction mixture was stirred at room temperature for $30 \mathrm{~min}$. The organic phase was washed three times with saturated $\mathrm{NaHCO}_{3}$, dried over $\mathrm{MgSO}_{4}$, filtered, and evaporated. The residue was dissolved in $100 \mathrm{~mL}$ of $\mathrm{CH}_{2} \mathrm{Cl}_{2}$, and gaseous $\mathrm{NH}_{3}$ was bubbled through the solution for $15 \mathrm{~min}$ at $-10^{\circ} \mathrm{C}$. The mixture was stirred at room temperature overnight, then filtered over Celite and evaporated. The residue was dissolved with AcOEt and washed with water and brine. The organic layer was dried over $\mathrm{MgSO}_{4}$, filtered, and evaporated to yield $2.95 \mathrm{~g}(67 \%)$ of the title compound. ${ }^{15}$

${ }^{1} \mathrm{H}$ NMR $\left(\mathrm{CDCl}_{3}, \mathrm{Me}_{4} \mathrm{Si}\right) \delta 0.08(\mathrm{~s}, 9 \mathrm{H}), 1.05-1.15(\mathrm{~m}, 2 \mathrm{H})$, $3.00-3.10(\mathrm{~m}, 2 \mathrm{H}), 4.97$ (large s, $2 \mathrm{H}) ;{ }^{13} \mathrm{C} \mathrm{NMR}\left(\mathrm{CDCl}_{3}, \mathrm{Me}_{4^{-}}\right.$ Si) $\delta-1.6,11.2,52.0$.

General Procedure for the Synthesis of $\beta$-Aminoesters 5: Conditions A. To a mixture ${ }^{8 \mathrm{~b}}$ of 2-(trimethylsilylethane)sulfonamide 2 (363 mg, $2 \mathrm{mmol}$ ), 3-hydroxyquinuclidine (38 $\mathrm{mg}, 0.30 \mathrm{mmol}$ ), and molecular sieves ( $4 \AA, 400 \mathrm{mg}, 200 \mathrm{mg} /$ mmol substrate) in $1 \mathrm{~mL}$ of 2-propanol was added aldehyde 3 ( $2 \mathrm{mmol})$, methyl acrylate $(186 \mathrm{mg}, 2.2 \mathrm{mmol})$, and $\mathrm{Ti}(\mathrm{OiPr})_{4}$ (12 $\mathrm{mg}, 0.04 \mathrm{mmol})$. The reaction mixture was stirred for the indicated time at $70{ }^{\circ} \mathrm{C}$ and filtered over Celite. The Celite was rinsed three times with 2-propanol. The solvent was evaporated and the residue was diluted with AcOEt, neutralized with aq $\mathrm{KHSO}_{4}(1 \%)$, and washed with saturated $\mathrm{NaHCO}_{3}$ and brine. The organic layer was dried over $\mathrm{MgSO}_{4}$, filtered, and evaporated. Silica gel chromatography ( $\mathrm{Et}_{2} \mathrm{O} /$ hexane) yielded $\beta$-aminoester $\mathbf{5}$.

Conditions B. To a solution of 2-(trimethylsilylethane)sulfonamide 2 (363 mg, $2 \mathrm{mmol}$ ) and DABCO (112 mg, $1 \mathrm{mmol}$ ) in $1 \mathrm{~mL}$ of 2-propanol was added aldehyde $\mathbf{3}(10 \mathrm{mmol})$ and methyl acrylate ( $861 \mathrm{mg}, 10 \mathrm{mmol})$. The reaction mixture was stirred for the indicated time at $70{ }^{\circ} \mathrm{C}$, evaporated, diluted with AcOEt, neutralized with aq $\mathrm{KHSO}_{4}(1 \%)$, and washed with saturated $\mathrm{NaHCO}_{3}$ and brine. The organic layer was dried over $\mathrm{MgSO}_{4}$, filtered, and evaporated. Silica gel chromatography (Et $\mathrm{t}_{2} \mathrm{O} /$ hexane) yielded $\beta$-aminoester $\mathbf{5}$.

(15) (a) Stien, D.; Anderson, G. T.; Chase, C. E.; Koh, Y.-H.; Weinreb, S. M. J. Am. Chem. Soc. 1999, 121, 9574-9579. (b) Parker, L. L.; Gowans, N. D.; Jones, S. W.; Robins, D. J. Tetrahedron 2003, 59 10165-10171.
Methyl 2-(Phenyl(2-(trimethylsilyl)ethylsulfonamido)methyl)acrylate (5a): Conditions A. The crude product was purified by silica gel chromatography $\left(\mathrm{Et}_{2} \mathrm{O} /\right.$ hexane $\left.=3 / 7\right)$ to yield $519 \mathrm{mg}(73 \%)$ of the title compound as a white solid.

Conditions B. The crude product was purified by silica gel chromatography $\left(\mathrm{Et}_{2} \mathrm{O} /\right.$ hexane $\left.=3 / 7\right)$ to yield $614 \mathrm{mg}(86 \%)$ of the title compound as a white solid.

Mp 91.6-92.2 ${ }^{\circ} \mathrm{C}$; IR 3368 (m), 2955 (m), 1719 (s), 1335 (s) $\mathrm{cm}^{-1} ;{ }^{1} \mathrm{H} \mathrm{NMR}\left(\mathrm{CDCl}_{3}, \mathrm{Me}_{4} \mathrm{Si}\right) \delta-0.01(\mathrm{~s}, 9 \mathrm{H}), 0.80-1.05(\mathrm{~m}$, $2 \mathrm{H}), 2.75-2.95(\mathrm{~m}, 2 \mathrm{H}), 3.73(\mathrm{~s}, 3 \mathrm{H}), 5.45\left(\mathrm{~d}, 1 \mathrm{H}, J_{3}=9.3 \mathrm{~Hz}\right)$, $5.53\left(\mathrm{~d}, 1 \mathrm{H}, J_{3}=9.3 \mathrm{~Hz}\right), 6.02(\mathrm{~s}, 1 \mathrm{H}), 6.43\left(\mathrm{~d}, 1 \mathrm{H}, J_{4}=0.6\right.$ $\mathrm{Hz}), 7.25-7.45(\mathrm{~m}, 5 \mathrm{H}) ;{ }^{13} \mathrm{C} \mathrm{NMR}\left(\mathrm{CDCl}_{3}, \mathrm{Me}_{4} \mathrm{Si}\right) \delta-1.6,10.9$, 50.4, 52.6, 59.5, 127.0, 128.0, 128.4, 129.2, 139.6, 140.2, 166.3; ESIMS $m / z 174.8\left(\mathrm{M}-\mathrm{SESNH}_{2}+\mathrm{H}\right)^{+}, 356.0(\mathrm{M}+\mathrm{H})^{+}, 378.1$ $(\mathrm{M}+\mathrm{Na})^{+}, 710.9(2 \mathrm{M}+\mathrm{H})^{+}, 733.1(2 \mathrm{M}+\mathrm{Na})^{+} ; \mathrm{FAB}+\mathrm{m} / \mathrm{z}$ $175\left(\mathrm{M}-\mathrm{SESNH}_{2}+\mathrm{H}\right)^{+}, 356(\mathrm{M}+\mathrm{H})^{+}$; HRMS calcd for $\mathrm{C}_{16} \mathrm{H}_{26} \mathrm{NO}_{4} \mathrm{SSi} 356.1352$, found 356.1316.

Methyl 2-((3,5-Dimethoxyphenyl)(2-(trimethylsilyl)ethylsulfonamido)methyl)acrylate (5b): Conditions A. The crude product was purified by silica gel chromatography $\left(\mathrm{Et}_{2} \mathrm{O} /\right.$ hexane $\left.=4 / 6\right)$ to yield $383 \mathrm{mg}(46 \%)$ of the title compound as a white solid.

Conditions B. The crude product was purified by silica gel chromatography $\left(\mathrm{Et}_{2} \mathrm{O} /\right.$ hexane $\left.=4 / 6\right)$ to yield $557 \mathrm{mg}(67 \%)$ of the title compound as a white solid.

Mp 91.4-95.5 ${ }^{\circ} \mathrm{C}$; IR $3383(\mathrm{~m}), 2963(\mathrm{~m}), 1719(\mathrm{~s}), 1334(\mathrm{~s})$ $\mathrm{cm}^{-1} ;{ }^{1} \mathrm{H} \mathrm{NMR}\left(\mathrm{CDCl}_{3}, \mathrm{Me}_{4} \mathrm{Si}\right) \delta-0.01(\mathrm{~s}, 9 \mathrm{H}), 0.85-1.05(\mathrm{~m}$, $2 \mathrm{H}), 2.80-2.95(\mathrm{~m}, 2 \mathrm{H}), 3.74(\mathrm{~s}, 3 \mathrm{H}), 3.79(\mathrm{~s}, 6 \mathrm{H}), 5.36(\mathrm{~d}, 1 \mathrm{H}$, $\left.J_{3}=9.3 \mathrm{~Hz}\right), 5.55\left(\mathrm{~d}, 1 \mathrm{H}, J_{3}=9.3 \mathrm{~Hz}\right), 5.99(\mathrm{~s}, 1 \mathrm{H}), 6.35-6.40$ $(\mathrm{m}, 1 \mathrm{H}), 6.41\left(\mathrm{~d}, 1 \mathrm{H}, J_{4}=0.4 \mathrm{~Hz}\right), 6.50-6.55(\mathrm{~m}, 2 \mathrm{H}) ;{ }^{13} \mathrm{C} \mathrm{NMR}$ $\left(\mathrm{CDCl}_{3}, \mathrm{Me}_{4} \mathrm{Si}\right) \delta-1.7,10.9,50.4,52.6,55.8,59.5,99.9,105.2$, $128.1,139.9,142.0,161.4,166.3$; ESIMS $\mathrm{m} / \mathrm{z} 235.2(\mathrm{M}-$ $\left.\mathrm{SESNH}_{2}+\mathrm{H}\right)^{+}, 416.2(\mathrm{M}+\mathrm{H})^{+}, 831.3(2 \mathrm{M}+\mathrm{H})^{+}, 853.4(2 \mathrm{M}$ $+\mathrm{Na})^{+} ; \mathrm{FAB}+m / z 235\left(\mathrm{M}-\mathrm{SESNH}_{2}+\mathrm{H}\right)^{+}, 415\left(\mathrm{M}-\mathrm{e}^{-}\right)^{+}$ HRMS calcd for $\mathrm{C}_{18} \mathrm{H}_{29} \mathrm{NO}_{6} \mathrm{SSi} 415.1485$, found 415.1480 .

Methyl 4-(2-(Methoxycarbonyl)-1-(2-(trimethylsilyl)ethylsulfonamido)allyl)benzoate (5c): Conditions $A$. The crude product was purified by silica gel chromatography $\left(\mathrm{Et}_{2} \mathrm{O} /\right.$ hexane $=4 / 6)$ to yield $530 \mathrm{mg}(64 \%)$ of the title compound as a white solid.

Conditions B. The crude product was purified by silica gel chromatography $\left(\mathrm{Et}_{2} \mathrm{O} /\right.$ hexane $\left.=4 / 6\right)$ to yield $747 \mathrm{mg}(90 \%)$ of the title compound as a white solid.

Mp 103.7-108.5 ${ }^{\circ} \mathrm{C}$; IR 3406 (m), 2950 (m), 1719 (s), 1332 (s) $\mathrm{cm}^{-1} ;{ }^{1} \mathrm{H}$ NMR $\left(\mathrm{CDCl}_{3}, \mathrm{Me}_{4} \mathrm{Si}\right) \delta 0.00(\mathrm{~s}, 9 \mathrm{H}), 0.75-1.10$ $(\mathrm{m}, 2 \mathrm{H}), 2.85-3.00(\mathrm{~m}, 2 \mathrm{H}), 3.72(\mathrm{~s}, 3 \mathrm{H}), 3.93(\mathrm{~s}, 3 \mathrm{H}), 5.48(\mathrm{~d}$, $\left.1 \mathrm{H}, J_{3}=9.7 \mathrm{~Hz}\right), 5.71\left(\mathrm{~d}, 1 \mathrm{H}, J_{3}=9.7 \mathrm{~Hz}\right), 6.03(\mathrm{~s}, 1 \mathrm{H}), 6.46$ $(\mathrm{s}, 1 \mathrm{H}), 7.46\left(\mathrm{~d}, 2 \mathrm{H}, J_{3}=8.5 \mathrm{~Hz}\right), 8.03\left(\mathrm{~d}, 2 \mathrm{H}, J_{3}=8.5 \mathrm{~Hz}\right) ;{ }^{13} \mathrm{C}$ $\mathrm{NMR}\left(\mathrm{CDCl}_{3}, \mathrm{Me}_{4} \mathrm{Si}\right) \delta-1.6,10.9,50.6,52.6,52.7,59.5,126.8$, $128.9,130.2,130.4,139.5,144.6,166.1,167.0 ;$ ESIMS $\mathrm{m} / 2$ $414.1(\mathrm{M}+\mathrm{H})^{+}, 827.5(2 \mathrm{M}+\mathrm{H})^{+}, 849.3(2 \mathrm{M}+\mathrm{Na})^{+}$; FAB + $\mathrm{m} / \mathrm{z} 233\left(\mathrm{M}-\mathrm{SESNH}_{2}+\mathrm{H}\right)^{+}, 414(\mathrm{M}+\mathrm{H})^{+}$; HRMS calcd for $\mathrm{C}_{18} \mathrm{H}_{28} \mathrm{NO}_{6} \mathrm{SSi}$ 414.1407, found 414.1435.

Methyl 2-(Pyridin-3-yl(2-(trimethylsilyl)ethylsulfonamido)methyl)acrylate (5d): Conditions A. The crude product was purified by silica gel chromatography $\left(\mathrm{Et}_{2} \mathrm{O} /\right.$ hexane $\left.=4 / 6+1 \% \mathrm{Et}_{3} \mathrm{~N}\right)$ to yield $535 \mathrm{mg}(75 \%)$ of the title compound as a white solid.

Mp 129.2-129.8 ${ }^{\circ} \mathrm{C}$; IR 3418 (m), 2950 (m), 1716 (s), 1331 (s) $\mathrm{cm}^{-1} ;{ }^{1} \mathrm{H}$ NMR $\left(\mathrm{CDCl}_{3}, \mathrm{Me}_{4} \mathrm{Si}\right) \delta 0.02(\mathrm{~s}, 9 \mathrm{H}), 0.85-1.20$ $(\mathrm{m}, 2 \mathrm{H}), 2.80-3.05(\mathrm{~m}, 2 \mathrm{H}), 3.75(\mathrm{~s}, 3 \mathrm{H}), 5.48\left(\mathrm{~d}, 1 \mathrm{H}, J_{3}=9.5\right.$ $\mathrm{Hz}), 5.70\left(\mathrm{~d}, 1 \mathrm{H}, J_{3}=9.5 \mathrm{~Hz}\right), 6.07(\mathrm{~s}, 1 \mathrm{H}), 6.49\left(\mathrm{~d}, 1 \mathrm{H}, J_{4}=\right.$ $0.4 \mathrm{~Hz}), 7.32\left(\mathrm{dd}, 1 \mathrm{H}, J_{3}=8.0 \mathrm{~Hz}, J_{3}=4.8 \mathrm{~Hz}\right), 7.79(\mathrm{dt}, 1 \mathrm{H}$, $\left.J_{3}=8.0 \mathrm{~Hz}, J_{4}=1.7 \mathrm{~Hz}\right), 8.57\left(\mathrm{dd}, 1 \mathrm{H}, J_{3}=4.8 \mathrm{~Hz}, J_{4}=1.2\right.$ $\mathrm{Hz}), 8.61\left(\mathrm{~d}, 1 \mathrm{H}, J_{4}=1.9 \mathrm{~Hz}\right) ;{ }^{13} \mathrm{C} \mathrm{NMR}\left(\mathrm{CDCl}_{3}, \mathrm{Me}_{4} \mathrm{Si}\right) \delta-1.6$, $10.9,50.7,52.8,58.0,123.9,129.2,134.6,135.3,139.2,148.5$, 149.6, 166.0; ESIMS $\mathrm{m} / z$ 357.1 $(\mathrm{M}+\mathrm{H})^{+}, 713.3(2 \mathrm{M}+\mathrm{H})^{+}$; $\mathrm{FAB}+\mathrm{m} / z 357(\mathrm{M}+\mathrm{H})^{+}, 379(\mathrm{M}+\mathrm{Na})^{+}$; HRMS calcd for $\mathrm{C}_{17} \mathrm{H}_{27} \mathrm{~N}_{2} \mathrm{O}_{4} \mathrm{SSi}$ 357.1304, found 357.1301.

Methyl 2-((3-Fluorophenyl)(2-(trimethylsilyl)ethylsulfonamido)methyl)acrylate (5e): Conditions $\mathbf{A}$. The crude product was purified by silica gel chromatography $\left(\mathrm{Et}_{2} \mathrm{O} /\right.$ 
hexane $=3 / 7)$ to yield $269 \mathrm{mg}(36 \%)$ of the title compound as a white solid.

Conditions B. The crude product was purified by silica gel chromatography $\left(\mathrm{Et}_{2} \mathrm{O} /\right.$ hexane $\left.=3 / 7\right)$ to yield $592 \mathrm{mg}(79 \%)$ of the title compound as a white solid.

Mp 68.1-69.1 ${ }^{\circ} \mathrm{C}$; IR 3370 (m), 2958 (m), $1714(\mathrm{~s}), 1334$ (s), $1093(\mathrm{~m}) \mathrm{cm}^{-1} ;{ }^{1} \mathrm{H} \mathrm{NMR}\left(\mathrm{CDCl}_{3}, \mathrm{Me}_{4} \mathrm{Si}\right) \delta 0.01(\mathrm{~s}, 9 \mathrm{H}), 0.80-$ $1.10(\mathrm{~m}, 2 \mathrm{H}), 2.80-3.00(\mathrm{~m}, 2 \mathrm{H}), 3.75(\mathrm{~s}, 3 \mathrm{H}), 5.43\left(\mathrm{~d}, 1 \mathrm{H}, J_{3}\right.$ $=9.5 \mathrm{~Hz}), 5.62\left(\mathrm{~d}, 1 \mathrm{H}, J_{3}=9.5 \mathrm{~Hz}\right), 6.02(\mathrm{~s}, 1 \mathrm{H}), 6.45(\mathrm{~s}, 1 \mathrm{H})$, 6.95-7.25 (m, 3H), 7.30-7.45 (m, $1 \mathrm{H}) ;{ }^{13} \mathrm{C} \mathrm{NMR}\left(\mathrm{CDCl}_{3}, \mathrm{Me}_{4^{-}}\right.$ Si) $\delta-1.7,10.9,50.6,52.7,59.2\left(\mathrm{~d}, J_{4}=1.5 \mathrm{~Hz}\right), 114.0\left(\mathrm{~d}, J_{2}\right.$ $=22.6 \mathrm{~Hz}), 115.3\left(\mathrm{~d}, J_{2}=21.1 \mathrm{~Hz}\right), 122.5\left(\mathrm{~d}, J_{4}=2.8 \mathrm{~Hz}\right)$, 128.7, $130.7\left(\mathrm{~d}, J_{3}=8.5 \mathrm{~Hz}\right), 139.6,142.3\left(\mathrm{~d}, J_{3}=6.8 \mathrm{~Hz}\right)$, $163.3\left(\mathrm{~d}, J_{1}=246.9 \mathrm{~Hz}\right), 166.1 ;$ ESIMS $m / z 374.1(\mathrm{M}+\mathrm{H})^{+}$, $747.2(2 \mathrm{M}+\mathrm{H})^{+} ; \mathrm{FAB}+m / z 193\left(\mathrm{M}-\mathrm{SESNH}_{2}+\mathrm{H}\right)^{+}, 374(\mathrm{M}$ $+\mathrm{H})^{+}, 396(\mathrm{M}+\mathrm{Na})^{+}$; HRMS calcd for $\mathrm{C}_{16} \mathrm{H}_{25} \mathrm{FNO}_{4} \mathrm{SSi}$ 374.1258 , found 374.1273 .

Methyl 2-((2-Bromophenyl)(2-(trimethylsilyl)ethylsulfonamido)methyl)acrylate (5f): Conditions $A$. The crude product was purified by silica gel chromatography $\left(\mathrm{Et}_{2} \mathrm{O} /\right.$ hexane $=3 / 7)$ to yield $367 \mathrm{mg}(42 \%)$ of the title compound as a white solid.

Conditions B. The crude product was purified by silica gel chromatography $\left(\mathrm{Et}_{2} \mathrm{O} /\right.$ hexane $\left.=3 / 7\right)$ to yield $759 \mathrm{mg}(86 \%)$ of the title compound as a white solid.

Mp 104.4-105.3 ${ }^{\circ} \mathrm{C}$; IR 3378 (m), 2955 (m), 1726 (s), 1336 (s) $\mathrm{cm}^{-1} ;{ }^{1} \mathrm{H}$ NMR $\left(\mathrm{CDCl}_{3}, \mathrm{Me}_{4} \mathrm{Si}\right) \delta 0.00(\mathrm{~s}, 9 \mathrm{H}), 0.80-1.00$ $(\mathrm{m}, 2 \mathrm{H}), 2.85-3.10(\mathrm{~m}, 2 \mathrm{H}), 3.75(\mathrm{~s}, 3 \mathrm{H}), 5.32\left(\mathrm{~d}, 1 \mathrm{H}, J_{3}=8.9\right.$ $\mathrm{Hz}), 5.88\left(\mathrm{~d}, 1 \mathrm{H}, J_{3}=8.9 \mathrm{~Hz}\right), 6.03\left(\mathrm{~d}, 1 \mathrm{H}, J_{4}=1.5 \mathrm{~Hz}\right), 6.46$ $(\mathrm{s}, 1 \mathrm{H}), 7.20\left(\mathrm{td}, 1 \mathrm{H}, J_{3}=7.7 \mathrm{~Hz}, J_{4}=1.7 \mathrm{~Hz}\right), 7.37\left(\mathrm{td}, 1 \mathrm{H}, J_{3}\right.$ $\left.=7.7 \mathrm{~Hz}, J_{4}=1.3 \mathrm{~Hz}\right), 7.55\left(\mathrm{dd}, 1 \mathrm{H}, J_{3}=7.8 \mathrm{~Hz}, J_{4}=1.7\right.$ $\mathrm{Hz}), 7.60\left(\mathrm{dd}, 1 \mathrm{H}, J_{3}=7.8 \mathrm{~Hz}, J_{4}=1.3 \mathrm{~Hz}\right) ;{ }^{13} \mathrm{C} \mathrm{NMR}\left(\mathrm{CDCl}_{3}\right.$, $\left.\mathrm{Me}_{4} \mathrm{Si}\right) \delta-1.6,10.9,50.4,52.6,58.0,123.7,128.3,128.9,129.3$, $130.0,133.9,138.9,139.3,166.3 ;$ ESIMS $\mathrm{m} / z$ 434.0/436.0 (M $+\mathrm{H})^{+}, 867.1 / 869.1 / 871.1(2 \mathrm{M}+\mathrm{H})^{+} ; \mathrm{FAB}+\mathrm{m} / z$ 253/255 $(\mathrm{M}-$ $\left.\mathrm{SESNH}_{2}+\mathrm{H}\right)^{+}, 434 / 436(\mathrm{M}+\mathrm{H})^{+}, 456 / 458(\mathrm{M}+\mathrm{Na})^{+} ; \mathrm{HRMS}$ calcd for $\mathrm{C}_{16} \mathrm{H}_{25} \mathrm{BrNO}_{4} \mathrm{SSi}$ 434.0457, found 434.0442.

Methyl 2-(Naphthalen-2-yl(2-(trimethylsilyl)ethylsulfonamido)methyl)acrylate (5g): Conditions B. The crude product was purified by silica gel chromatography $\left(\mathrm{Et}_{2} \mathrm{O} /\right.$ hexane $=3 / 7)$ to yield $487 \mathrm{mg}(60 \%)$ of the title compound as a white solid.

Mp 110.7-112.0 ${ }^{\circ} \mathrm{C}$; IR 3388 (m), 2963 (m), 1715 (s), 1333 (s) $\mathrm{cm}^{-1} ;{ }^{1} \mathrm{H} \mathrm{NMR}\left(\mathrm{CDCl}_{3}, \mathrm{Me}_{4} \mathrm{Si}\right) \delta-0.07(\mathrm{~s}, 9 \mathrm{H}), 0.85-1.00$ (m, 2H), 2.80-2.95 (m, 2H), $3.72(\mathrm{~s}, 3 \mathrm{H}), 5.63\left(\mathrm{~d}, 1 \mathrm{H}, J_{3}=9.3\right.$ $\mathrm{Hz}), 5.70\left(\mathrm{~d}, 1 \mathrm{H}, J_{3}=9.3 \mathrm{~Hz}\right), 6.09(\mathrm{~s}, 1 \mathrm{H}), 6.48(\mathrm{~s}, 1 \mathrm{H}), 7.45-$ $7.55(\mathrm{~m}, 3 \mathrm{H}), 7.75-7.95(\mathrm{~m}, 4 \mathrm{H}) ;{ }^{13} \mathrm{C} \mathrm{NMR}\left(\mathrm{CDCl}_{3}, \mathrm{Me}_{4} \mathrm{Si}\right) \delta$ $-1.7,10.9,50.5,52.6,59.6,124.9,126.0,126.8,126.9,128.0$, 128.1, 128.5, 129.1, 133.3, 133.6, 136.9, 140.1, 166.3; ESIMS $m / z 225.1\left(\mathrm{M}-\mathrm{SESNH}_{2}+\mathrm{H}\right)^{+}, 405.9(\mathrm{M}+\mathrm{H})^{+} ; \mathrm{FAB}+m / z$ $225\left(\mathrm{M}-\mathrm{SESNH}_{2}+\mathrm{H}\right)^{+}, 405(\mathrm{M}+\mathrm{H})^{+}$; HRMS calcd for $\mathrm{C}_{20} \mathrm{H}_{27} \mathrm{NO}_{4} \mathrm{SSi}$ 405.1430, found 405.1431.

Methyl 2-(m-Tolyl(2-(trimethylsilyl)ethylsulfonamido)methyl)acrylate (5h): Conditions $B$. The crude product was purified by silica gel chromatography $\left(\mathrm{Et}_{2} \mathrm{O} /\right.$ hexane $\left.=3 / 7\right)$ to yield $633 \mathrm{mg}(86 \%)$ of the title compound as a white solid.

$\mathrm{Mp} 60.7-62.4{ }^{\circ} \mathrm{C}$; IR $3425(\mathrm{~m}), 2955(\mathrm{~m}), 1720(\mathrm{~s}), 1328(\mathrm{~s})$ $\mathrm{cm}^{-1} ;{ }^{1} \mathrm{H} \mathrm{NMR}\left(\mathrm{CDCl}_{3}, \mathrm{Me}_{4} \mathrm{Si}\right) \delta-0.01(\mathrm{~s}, 9 \mathrm{H}), 0.80-1.00(\mathrm{~m}$, $2 \mathrm{H}), 2.36(\mathrm{~s}, 3 \mathrm{H}), 2.75-2.95(\mathrm{~m}, 2 \mathrm{H}), 3.73(\mathrm{~s}, 3 \mathrm{H}), 5.41(\mathrm{~d}, 1 \mathrm{H}$, $\left.J_{3}=9.3 \mathrm{~Hz}\right), 5.54\left(\mathrm{~d}, 1 \mathrm{H}, J_{3}=9.3 \mathrm{~Hz}\right), 6.02(\mathrm{~s}, 1 \mathrm{H}), 6.42(\mathrm{~s}$, $1 \mathrm{H}), 7.05-7.35(\mathrm{~m}, 4 \mathrm{H}) ;{ }^{13} \mathrm{C} \mathrm{NMR}\left(\mathrm{CDCl}_{3}, \mathrm{Me}_{4} \mathrm{Si}\right) \delta-1.7,10.9$, 21.9, 50.4, 52.6, 59.4, 124.0, 127.7, 127.8, 129.1, 129.2, 138.9, 139.5, 140.2, 166.3; ESIMS $m / z 189.1\left(\mathrm{M}-\mathrm{SESNH}_{2}+\mathrm{H}\right)^{+}$, $370.1(\mathrm{M}+\mathrm{H})^{+}, 739.3(2 \mathrm{M}+\mathrm{H})^{+}, 761.2(2 \mathrm{M}+\mathrm{Na})^{+} ; \mathrm{FAB}+$ $\mathrm{m} / z 189\left(\mathrm{M}-\mathrm{SESNH}_{2}+\mathrm{H}\right)^{+}, 370(\mathrm{M}+\mathrm{H})^{+}, 392(\mathrm{M}+\mathrm{Na})^{+}$; HRMS calcd for $\mathrm{C}_{17} \mathrm{H}_{28} \mathrm{NO}_{4} \mathrm{SSi} 370.1508$, found 370.1548.

Methyl 2-((3,5-Dimethylphenyl)(2-(trimethylsilyl)ethylsulfonamido)methyl)acrylate (5i): Conditions B. The crude product was purified by silica gel chromatography $\left(\mathrm{Et}_{2} \mathrm{O} /\right.$ hexane $=3 / 7)$ to yield $537 \mathrm{mg}(70 \%)$ of the title compound as a white solid.
Mp 92.0-93.4 ${ }^{\circ} \mathrm{C}$; IR 3383 (m), 2953 (m), 1715 (s), 1334 (s) $\mathrm{cm}^{-1} ;{ }^{1} \mathrm{H}$ NMR $\left(\mathrm{CDCl}_{3}, \mathrm{Me}_{4} \mathrm{Si}\right) \delta-0.01(\mathrm{~s}, 9 \mathrm{H}), 0.85-1.00(\mathrm{~m}$, $2 \mathrm{H}), 2.32(\mathrm{~s}, 6 \mathrm{H}), 2.80-2.95(\mathrm{~m}, 2 \mathrm{H}), 3.74(\mathrm{~s}, 3 \mathrm{H}), 5.36(\mathrm{~d}, 1 \mathrm{H}$, $\left.J_{3}=9.3 \mathrm{~Hz}\right), 5.42\left(\mathrm{~d}, 1 \mathrm{H}, J_{3}=9.3 \mathrm{~Hz}\right), 6.02(\mathrm{~s}, 1 \mathrm{H}), 6.42(\mathrm{~d}$, $\left.1 \mathrm{H}, J_{4}=0.6 \mathrm{~Hz}\right), 6.94(\mathrm{~s}, 1 \mathrm{H}), 6.97(\mathrm{~s}, 2 \mathrm{H}) ;{ }^{13} \mathrm{C} \mathrm{NMR}\left(\mathrm{CDCl}_{3}\right.$, $\left.\mathrm{Me}_{4} \mathrm{Si}\right) \delta-1.7,10.9,21.8,50.3,52.6,59.4,124.7,127.8,130.1$, 138.8, 139.4, 140.2, 166.3; ESIMS $m / z 203.3\left(\mathrm{M}-\mathrm{SESNH}_{2}+\right.$ $\mathrm{H})^{+}, 384.3(\mathrm{M}+\mathrm{H})^{+}, 767.3(2 \mathrm{M}+\mathrm{H})^{+} ; \mathrm{FAB}+\mathrm{m} / z 203(\mathrm{M}-$ $\left.\mathrm{SESNH}_{2}+\mathrm{H}\right)^{+}, 384(\mathrm{M}+\mathrm{H})^{+}, 406(\mathrm{M}+\mathrm{Na})^{+}$; HRMS calcd for $\mathrm{C}_{18} \mathrm{H}_{30} \mathrm{NO}_{4} \mathrm{SSi} 384.1665$, found 384.1670.

Methyl 2-((2,3-Methylenedioxyphenyl)(2-(trimethylsilyl)ethylsulfonamido)methyl)acrylate (5j): Conditions B. The crude product was purified by silica gel chromatography $\left(\mathrm{Et}_{2} \mathrm{O} /\right.$ hexane $\left.=4 / 6\right)$ to yield $526 \mathrm{mg}(66 \%)$ of the title compound as a white solid.

Mp 93.0-94.8 ${ }^{\circ} \mathrm{C}$; IR 3375 (m), 2955 (m), 1725 (s), 1339 (s) $\mathrm{cm}^{-1} ;{ }^{1} \mathrm{H} \mathrm{NMR}\left(\mathrm{CDCl}_{3}, \mathrm{Me}_{4} \mathrm{Si}\right) \delta-0.02(\mathrm{~s}, 9 \mathrm{H}), 0.75-1.05(\mathrm{~m}$, $2 \mathrm{H}), 2.75-3.05(\mathrm{~m}, 2 \mathrm{H}), 3.76(\mathrm{~s}, 3 \mathrm{H}), 5.52\left(\mathrm{~d}, 1 \mathrm{H}, J_{3}=9.8 \mathrm{~Hz}\right)$, $5.57\left(\mathrm{~d}, 1 \mathrm{H}, J_{3}=9.8 \mathrm{~Hz}\right), 5.99(\mathrm{~s}, 1 \mathrm{H}), 6.01(\mathrm{~s}, 1 \mathrm{H}), 6.38(\mathrm{~s}$, $1 \mathrm{H}), 6.75-6.95(\mathrm{~m}, 3 \mathrm{H}) ;{ }^{13} \mathrm{C} \mathrm{NMR}\left(\mathrm{CDCl}_{3}, \mathrm{Me}_{4} \mathrm{Si}\right) \delta-1.7,10.8$, $50.2,52.6,54.8,101.6,108.9,120.9,121.5,122.5,127.4,139.2$, 144.9, 148.0, 166.2; ESIMS $m / z 219.2\left(\mathrm{M}-\mathrm{SESNH}_{2}+\mathrm{H}\right)^{+}$, $400.0(\mathrm{M}+\mathrm{H})^{+}, 799.2(2 \mathrm{M}+\mathrm{H})^{+}, 821.2(2 \mathrm{M}+\mathrm{Na})^{+} ; \mathrm{FAB}+$ $m / z 219\left(\mathrm{M}-\mathrm{SESNH}_{2}+\mathrm{H}\right)^{+}, 399\left(\mathrm{M}-\mathrm{e}^{-}\right)^{+}, 422(\mathrm{M}+\mathrm{Na})^{+}$; HRMS calcd for $\mathrm{C}_{17} \mathrm{H}_{25} \mathrm{NO}_{6} \mathrm{SSi} 399.1172$, found 399.1164.

Methyl 2-((4-Chlorophenyl)(2-(trimethylsilyl)ethylsulfonamido)methyl)acrylate (5k): Conditions $B$. The crude product was purified by silica gel chromatography $\left(\mathrm{Et}_{2} \mathrm{O} /\right.$ hexane $=3 / 7)$ to yield $670 \mathrm{mg}(86 \%)$ of the title compound as a white solid.

Mp 106.4-107.1 ${ }^{\circ} \mathrm{C}$; IR 3425 (m), 1713 (s), 1335 (s) $\mathrm{cm}^{-1}$; ${ }^{1} \mathrm{H}$ NMR $\left(\mathrm{CDCl}_{3}, \mathrm{Me}_{4} \mathrm{Si}\right) \delta 0.02(\mathrm{~s}, 9 \mathrm{H}), 0.80-1.10(\mathrm{~m}, 2 \mathrm{H})$, $2.80-3.00(\mathrm{~m}, 2 \mathrm{H}), 3.74(\mathrm{~s}, 3 \mathrm{H}), 5.41\left(\mathrm{~d}, 1 \mathrm{H}, J_{3}=9.3 \mathrm{~Hz}\right), 5.54$ $\left(\mathrm{d}, 1 \mathrm{H}, J_{3}=9.3 \mathrm{~Hz}\right), 6.01(\mathrm{~s}, 1 \mathrm{H}), 6.44(\mathrm{~s}, 1 \mathrm{H}), 7.34(\mathrm{~s}, 4 \mathrm{H}) ;{ }^{13} \mathrm{C}$ $\mathrm{NMR}\left(\mathrm{CDCl}_{3}, \mathrm{Me}_{4} \mathrm{Si}\right) \delta-1.6,10.9,50.6,52.7,59.2,128.3,128.5$, 129.3, 134.3, 138.1, 139.7, 166.1; ESIMS $\mathrm{m} / z$ 208.8 (M $\left.\mathrm{SESNH}_{2}+\mathrm{H}\right)^{+}, 390.1(\mathrm{M}+\mathrm{H})^{+}, 412.0(\mathrm{M}+\mathrm{Na})^{+}, 779.0(2 \mathrm{M}$ $+\mathrm{H})^{+}, 801.1(2 \mathrm{M}+\mathrm{Na})^{+} ; \mathrm{FAB}+\mathrm{m} / z 209\left(\mathrm{M}-\mathrm{SESNH}_{2}+\right.$ $\mathrm{H})^{+}, 390(\mathrm{M}+\mathrm{H})^{+}, 412(\mathrm{M}+\mathrm{Na})^{+}$; HRMS calcd for $\mathrm{C}_{16} \mathrm{H}_{25^{-}}$ $\mathrm{ClNO}_{4} \mathrm{SSi} 390.0962$, found 390.0974 .

Methyl 2-((2-Iodophenyl)(2-(trimethylsilyl)ethylsulfonamido)methyl)acrylate $(5 l)$ : Conditions $B$. The crude product was purified by silica gel chromatography $\left(\mathrm{Et}_{2} \mathrm{O} /\right.$ hexane $=3 / 7)$ to yield $707 \mathrm{mg}(73 \%)$ of the title compound as a white solid.

Mp 127.4-128.6 ${ }^{\circ} \mathrm{C}$; IR 3383 (m), 2953 (m), 1723 (s), 1346 (s) $\mathrm{cm}^{-1} ;{ }^{1} \mathrm{H}$ NMR $\left(\mathrm{CDCl}_{3}, \mathrm{Me}_{4} \mathrm{Si}\right) \delta 0.01(\mathrm{~s}, 9 \mathrm{H}), 0.80-1.05$ $(\mathrm{m}, 2 \mathrm{H}), 2.85-3.15(\mathrm{~m}, 2 \mathrm{H}), 3.77(\mathrm{~s}, 3 \mathrm{H}), 5.21\left(\mathrm{~d}, 1 \mathrm{H}, J_{3}=8.7\right.$ $\mathrm{Hz}), 5.78\left(\mathrm{~d}, 1 \mathrm{H}, J_{3}=8.7 \mathrm{~Hz}\right), 6.02\left(\mathrm{~d}, 1 \mathrm{H}, J_{4}=0.9 \mathrm{~Hz}\right), 6.47$ $(\mathrm{s}, 1 \mathrm{H}), 7.03\left(\mathrm{ddd}, 1 \mathrm{H}, J_{3}=8.0 \mathrm{~Hz}, J_{3}=7.2 \mathrm{~Hz}, J_{4}=1.9 \mathrm{~Hz}\right.$ ), $7.40\left(\mathrm{ddd}, 1 \mathrm{H}, J_{3}=7.8 \mathrm{~Hz}, J_{3}=7.2 \mathrm{~Hz}, J_{4}=1.3 \mathrm{~Hz}\right), 7.51(\mathrm{dd}$, $\left.1 \mathrm{H}, J_{3}=7.8 \mathrm{~Hz}, J_{4}=1.9 \mathrm{~Hz}\right), 7.90\left(\mathrm{dd}, 1 \mathrm{H}, J_{3}=7.9 \mathrm{~Hz}, J_{4}=\right.$ $1.2 \mathrm{~Hz}) ;{ }^{13} \mathrm{C} \mathrm{NMR}\left(\mathrm{CDCl}_{3}, \mathrm{Me}_{4} \mathrm{Si}\right) \delta-1.5,10.9,50.6,52.6,62.3$, 99.8, 128.7, 129.0, 129.2, 130.2, 139.6, 140.7, 142.0, 166.3; ESIMS $m / z 300.9\left(\mathrm{M}-\mathrm{SESNH}_{2}+\mathrm{H}\right)^{+}, 482.1(\mathrm{M}+\mathrm{H})^{+}$; FAB + $\mathrm{m} / \mathrm{z} 301\left(\mathrm{M}-\mathrm{SESNH}_{2}+\mathrm{H}\right)^{+}, 482(\mathrm{M}+\mathrm{H})^{+}, 504(\mathrm{M}+\mathrm{Na})^{+}$; HRMS calcd for $\mathrm{C}_{16} \mathrm{H}_{25} \mathrm{INO}_{4} \mathrm{SSi} 482.0318$, found 482.0311 .

Methyl 2-((2-(Trimethylsilyl)ethylsulfonamido)(4-(2(trimethylsilyl)ethynyl)phenyl)methyl)acrylate (5m): Conditions B. The crude product was purified by silica gel chromatography $\left(\mathrm{Et}_{2} \mathrm{O} /\right.$ hexane $\left.=3 / 7\right)$ to yield $551 \mathrm{mg}(61 \%)$ of the title compound as a white solid.

Mp 90.2-91.4 ${ }^{\circ} \mathrm{C}$; IR 3380 (m), 2955 (m), 2158 (m), 1719 (s), $1334(\mathrm{~s}) \mathrm{cm}^{-1} ;{ }^{1} \mathrm{H} \mathrm{NMR}\left(\mathrm{CDCl}_{3}, \mathrm{Me}_{4} \mathrm{Si}\right) \delta 0.02(\mathrm{~s}, 9 \mathrm{H}), 0.26$ $(\mathrm{s}, 9 \mathrm{H}), 0.75-1.10(\mathrm{~m}, 2 \mathrm{H}), 2.80-3.00(\mathrm{~m}, 2 \mathrm{H}), 3.72(\mathrm{~s}, 3 \mathrm{H})$, $5.42\left(\mathrm{~d}, 1 \mathrm{H}, J_{3}=9.5 \mathrm{~Hz}\right), 5.53\left(\mathrm{~d}, 1 \mathrm{H}, J_{3}=9.5 \mathrm{~Hz}\right), 6.01(\mathrm{~s}$, $1 \mathrm{H}), 6.43(\mathrm{~s}, 1 \mathrm{H}), 7.31\left(\mathrm{~d}, 2 \mathrm{H}, J_{3}=8.5 \mathrm{~Hz}\right), 7.46\left(\mathrm{~d}, 2 \mathrm{H}, J_{3}=\right.$ $8.5 \mathrm{~Hz}) ;{ }^{13} \mathrm{C} \mathrm{NMR}\left(\mathrm{CDCl}_{3}, \mathrm{Me}_{4} \mathrm{Si}\right) \delta-1.6,0.3,10.9,50.6,52.6$, 59.4, 95.3, 104.8, 123.3, 126.8, 128.5, 132.7, 139.7, 139.8, 166.1; ESIMS $m / z 271.3\left(\mathrm{M}-\mathrm{SESNH}_{2}+\mathrm{H}\right)^{+}, 452.0(\mathrm{M}+\mathrm{H})^{+} ; \mathrm{FAB}+$ $\mathrm{m} / z 271\left(\mathrm{M}-\mathrm{SESNH}_{2}+\mathrm{H}\right)^{+}, 452(\mathrm{M}+\mathrm{H})^{+}, 474(\mathrm{M}+\mathrm{Na})^{+}$; HRMS calcd for $\mathrm{C}_{21} \mathrm{H}_{34} \mathrm{NO}_{4} \mathrm{SSi} 452.1747$, found 452.1755 . 
Methyl 2-((4-Nitrophenyl)(2-(trimethylsilyl)ethylsulfonamido)methyl)acrylate (5n): Conditions B. The crude product was purified by silica gel chromatography $\left(\mathrm{Et}_{2} \mathrm{O} /\right.$ hexane $=4 / 6)$ to yield $481 \mathrm{mg}(60 \%)$ of the title compound as a white solid.

Mp 104.9-113.7 ${ }^{\circ} \mathrm{C}$; IR 3368 (m), 2958 (m), 1715 (s), 1526 (s), $1354(\mathrm{~s}), 1339(\mathrm{~s}) \mathrm{cm}^{-1} ;{ }^{1} \mathrm{H} \mathrm{NMR}\left(\mathrm{CDCl}_{3}, \mathrm{Me}_{4} \mathrm{Si}\right) \delta 0.03(\mathrm{~s}$, $9 \mathrm{H}), 0.75-1.15(\mathrm{~m}, 2 \mathrm{H}), 2.85-3.05(\mathrm{~m}, 2 \mathrm{H}), 3.75(\mathrm{~s}, 3 \mathrm{H}), 5.51$ $\left(\mathrm{d}, 1 \mathrm{H}, J_{3}=9.8 \mathrm{~Hz}\right), 5.77\left(\mathrm{~d}, 1 \mathrm{H}, J_{3}=9.8 \mathrm{~Hz}\right), 6.07(\mathrm{~s}, 1 \mathrm{H})$, $6.51(\mathrm{~s}, 1 \mathrm{H}), 7.59\left(\mathrm{~d}, 2 \mathrm{H}, J_{3}=8.8 \mathrm{~Hz}\right), 8.22\left(\mathrm{~d}, 2 \mathrm{H}, J_{3}=8.8\right.$ $\mathrm{Hz}) ;{ }^{13} \mathrm{C} \mathrm{NMR}\left(\mathrm{CDCl}_{3}, \mathrm{Me}_{4} \mathrm{Si}\right) \delta-1.6,11.0,50.8,52.9,59.4$, $124.3,127.8,129.6,139.1,146.9,147.9,165.9 ;$ ESIMS $\mathrm{m} / \mathrm{z}$ $801.2(2 \mathrm{M}+\mathrm{H})^{+}, 823.3(2 \mathrm{M}+\mathrm{Na})^{+} ; \mathrm{FAB}+\mathrm{m} / z 401(\mathrm{M}+\mathrm{H})^{+}$, $423(\mathrm{M}+\mathrm{Na})^{+}$; HRMS calcd for $\mathrm{C}_{16} \mathrm{H}_{25} \mathrm{~N}_{2} \mathrm{O}_{6} \mathrm{SSi} 401.1203$, found 401.1187.

Methyl 2-(Furan-2-yl(2-(trimethylsilyl)ethylsulfonamido)methyl)acrylate (5o): Conditions B. The crude product was purified by silica gel chromatography $\left(\mathrm{Et}_{2} \mathrm{O} /\right.$ hexane $=3 / 7)$ to yield $489 \mathrm{mg}(71 \%)$ of the title compound as a yellow oil.

IR $3370(\mathrm{~m}), 2955(\mathrm{~m}), 1723(\mathrm{~s}), 1335(\mathrm{~s}) \mathrm{cm}^{-1} ;{ }^{1} \mathrm{H}$ NMR $\left(\mathrm{CDCl}_{3}, \mathrm{Me}_{4} \mathrm{Si}\right) \delta 0.02(\mathrm{~s}, 9 \mathrm{H}), 0.80-1.05(\mathrm{~m}, 2 \mathrm{H}), 2.80-3.00$ $(\mathrm{m}, 2 \mathrm{H}), 3.77(\mathrm{~s}, 3 \mathrm{H}), 5.49\left(\mathrm{~d}, 1 \mathrm{H}, J_{3}=9.5 \mathrm{~Hz}\right), 5.57\left(\mathrm{~d}, 1 \mathrm{H}, J_{3}\right.$ $=9.5 \mathrm{~Hz}), 6.00(\mathrm{~s}, 1 \mathrm{H}), 6.27\left(\mathrm{~d}, 1 \mathrm{H}, J_{3}=3.3 \mathrm{~Hz}\right), 6.34(\mathrm{dd}, 1 \mathrm{H}$, $\left.J_{3}=3.2 \mathrm{~Hz}, J_{3}=1.9 \mathrm{~Hz}\right), 6.42(\mathrm{~s}, 1 \mathrm{H}), 7.37\left(\mathrm{dd}, 1 \mathrm{H}, J_{3}=1.9\right.$ $\left.\mathrm{Hz}, J_{4}=1.0 \mathrm{~Hz}\right) ;{ }^{13} \mathrm{C} \mathrm{NMR}\left(\mathrm{CDCl}_{3}, \mathrm{Me}_{4} \mathrm{Si}\right) \delta-1.6,10.8,50.4$, 52.7, 53.58, 108.0, 111.2, 128.6, 138.2, 142.9, 152.3, 166.1; ESIMS $m / z 346.1(\mathrm{M}+\mathrm{H})^{+}, 691.3(2 \mathrm{M}+\mathrm{H})^{+}, 713.3(2 \mathrm{M}+$ $\mathrm{Na})^{+} ; \mathrm{FAB}+m / z 165\left(\mathrm{M}-\mathrm{SESNH}_{2}+\mathrm{H}\right)^{+}, 346(\mathrm{M}+\mathrm{H})^{+}, 368$ $(\mathrm{M}+\mathrm{Na})^{+}$; HRMS calcd for $\mathrm{C}_{14} \mathrm{H}_{24} \mathrm{NO}_{5} \mathrm{SSi} 346.1144$, found 346.1149 .

General Procedure for the Alkylation of $\beta$-Aminoesters 6. To a mixture ${ }^{5 \mathrm{a}}$ of $\beta$-aminoester $5(0.25 \mathrm{mmol})$ and $\mathrm{K}_{2} \mathrm{CO}_{3}(345 \mathrm{mg}, 2.5 \mathrm{mmol})$ in $3.5 \mathrm{~mL}$ of DMF was added allyl bromide (121 mg, $1 \mathrm{mmol}$ ). The mixture was stirred at room temperature for $6 \mathrm{~h}$, then filtered and diluted with AcOEt. The organic layer was successively washed with water and brine, dried over $\mathrm{MgSO}_{4}$, filtered, and evaporated, to yield the corresponding $N$-allyl- $\beta$-aminoester 6 .

Methyl 2-( $(N$-Allyl-2-(trimethylsilyl)ethan-3-ylsulfonamido)(phenyl)methyl)acrylate (6a). Alkylation of the $\beta$-aminoester 5a yielded $97 \mathrm{mg}(98 \%)$ of the title compound as a pale yellow oil.

IR $2953(\mathrm{~m}), 1720(\mathrm{~s}), 1328(\mathrm{~s}) \mathrm{cm}^{-1} ;{ }^{1} \mathrm{H} \mathrm{NMR}\left(\mathrm{CDCl}_{3}, \mathrm{Me}_{4}{ }^{-}\right.$ Si) $\delta 0.04(\mathrm{~s}, 9 \mathrm{H}), 0.95-1.15(\mathrm{~m}, 2 \mathrm{H}), 2.75-3.05(\mathrm{~m}, 2 \mathrm{H}), 3.70$ (s, 3H), $3.93\left(\mathrm{~d}, 2 \mathrm{H}, J_{3}=6.5 \mathrm{~Hz}\right), 4.95-5.15(\mathrm{~m}, 2 \mathrm{H}), 5.20-$ $5.45(\mathrm{~m}, 1 \mathrm{H}), 5.91\left(\mathrm{dd}, 1 \mathrm{H}, J_{2}=0.6 \mathrm{~Hz}, J_{4}=1.7 \mathrm{~Hz}\right), 6.09(\mathrm{~s}$, $1 \mathrm{H}), 6.58\left(\mathrm{dd}, 1 \mathrm{H}, J_{2}=0.6 \mathrm{~Hz}, J_{4}=1.3 \mathrm{~Hz}\right), 7.25-7.45(\mathrm{~m}$, $5 \mathrm{H}) ;{ }^{13} \mathrm{C} \mathrm{NMR}\left(\mathrm{CDCl}_{3}, \mathrm{Me}_{4} \mathrm{Si}\right) \delta-1.6,10.7,49.1,51.1,52.5$, $62.4,118.9,128.5,128.8,129.0,129.1,134.7,138.1,140.3$, 166.8; ESIMS $m / z$ 396.1 $(\mathrm{M}+\mathrm{H})^{+}, 791.7(2 \mathrm{M}+\mathrm{H})^{+}, 813.3$ $(2 \mathrm{M}+\mathrm{Na})^{+} ; \mathrm{FAB}+m / z 396(\mathrm{M}+\mathrm{H})^{+}, 418(\mathrm{M}+\mathrm{Na})^{+} ; \mathrm{HRMS}$ calcd for $\mathrm{C}_{19} \mathrm{H}_{30} \mathrm{NO}_{4} \mathrm{SSi} 396.1665$, found 356.1675 .

Methyl 2-( $(\mathrm{N}$-Allyl-2-(trimethylsilyl)ethan-3-ylsulfonamido)(3,5-dimethoxyphenyl)methyl)acrylate (6b). Alkylation of the $\beta$-aminoester $\mathbf{5 b}$ yielded $112 \mathrm{mg}$ (98\%) of the title compound as a pale yellow oil.

IR $2950(\mathrm{~m}), 1719(\mathrm{~s}), 1330(\mathrm{~s}) \mathrm{cm}^{-1} ;{ }^{1} \mathrm{H} \mathrm{NMR}\left(\mathrm{CDCl}_{3}, \mathrm{Me}_{4^{-}}\right.$ Si) $\delta 0.04(\mathrm{~s}, 9 \mathrm{H}), 0.95-1.15(\mathrm{~m}, 2 \mathrm{H}), 2.85-3.05(\mathrm{~m}, 2 \mathrm{H}), 3.73$ $(\mathrm{s}, 3 \mathrm{H}), 3.79(\mathrm{~s}, 6 \mathrm{H}), 3.93\left(\mathrm{~d}, 2 \mathrm{H}, J_{3}=6.7 \mathrm{~Hz}\right), 4.95-5.15(\mathrm{~m}$, $2 \mathrm{H}), 5.25-5.50(\mathrm{~m}, 1 \mathrm{H}), 5.89\left(\mathrm{~d}, 1 \mathrm{H}, J_{4}=1.3 \mathrm{~Hz}\right), 6.03(\mathrm{~s}, 1 \mathrm{H})$, $6.41\left(\mathrm{t}, 1 \mathrm{H}, J_{3}=2.2 \mathrm{~Hz}\right), 6.46\left(\mathrm{t}, 2 \mathrm{H}, J_{3}=2.2 \mathrm{~Hz}\right), 6.55(\mathrm{~d}, 1 \mathrm{H}$, $\left.J_{4}=0.7 \mathrm{~Hz}\right) ;{ }^{13} \mathrm{C} \mathrm{NMR}\left(\mathrm{CDCl}_{3}, \mathrm{Me}_{4} \mathrm{Si}\right) \delta-1.6,10.7,49.0,51.0$, 52.6, 55.7, 60.3, 100.2, 107.2, 118.9, 128.8, 134.9, 140.2, 140.5, 161.4, 166.8; ESIMS $m / z 456.2(\mathrm{M}+\mathrm{H})^{+}, 478.3(\mathrm{M}+\mathrm{Na})^{+}$, $911.5(2 \mathrm{M}+\mathrm{H})^{+} ; \mathrm{FAB}+\mathrm{m} / z 455\left(\mathrm{M}-\mathrm{e}^{-}\right)^{+}, 478(\mathrm{M}+\mathrm{Na})^{+}$; HRMS calcd for $\mathrm{C}_{21} \mathrm{H}_{33} \mathrm{NO}_{6} \mathrm{SSi} 455.1798$, found 455.1815 .

Methyl 4-(1-(N-Allyl-2-(trimethylsilyl)ethan-3-ylsulfonamido)-2-(methoxycarbonyl)allyl)benzoate (6c). Alkylation of the $\beta$-aminoester 5c yielded $110 \mathrm{mg} \mathrm{(97 \% )}$ of the title compound as a pale yellow oil.
IR $2955(\mathrm{~m}), 1719(\mathrm{~s}), 1329(\mathrm{~s}) \mathrm{cm}^{-1} ;{ }^{1} \mathrm{H} \mathrm{NMR}\left(\mathrm{CDCl}_{3}, \mathrm{Me}_{4}\right.$ Si) $\delta 0.05(\mathrm{~s}, 9 \mathrm{H}), 0.95-1.15(\mathrm{~m}, 2 \mathrm{H}), 2.80-3.10(\mathrm{~m}, 2 \mathrm{H}), 3.73$ $(\mathrm{s}, 3 \mathrm{H}), 3.85-4.00(\mathrm{~m}, 5 \mathrm{H}), 4.95-5.15(\mathrm{~m}, 2 \mathrm{H}), 5.25-5.50(\mathrm{~m}$, $1 \mathrm{H}), 5.85\left(\mathrm{~d}, 1 \mathrm{H}, J_{4}=1.7 \mathrm{~Hz}\right), 6.14(\mathrm{~s}, 1 \mathrm{H}), 6.62\left(\mathrm{~d}, 1 \mathrm{H}, J_{4}=\right.$ $1.1 \mathrm{~Hz}), 7.40\left(\mathrm{~d}, 2 \mathrm{H}, J_{3}=8.2 \mathrm{~Hz}\right), 8.05\left(\mathrm{~d}, 2 \mathrm{H}, J_{3}=8.4 \mathrm{~Hz}\right)$; ${ }^{13} \mathrm{C} \mathrm{NMR}\left(\mathrm{CDCl}_{3}, \mathrm{Me}_{4} \mathrm{Si}\right) \delta-1.6,10.7,49.1,51.2,52.6,52.7$, $62.0,119.3,128.9,129.8,130.2,130.3,134.4,139.7,143.6$, 166.6, 167.0; ESIMS $\mathrm{m} / z$ 454.2 $(\mathrm{M}+\mathrm{H})^{+}, 476.2(\mathrm{M}+\mathrm{Na})^{+}$, $907.6(2 \mathrm{M}+\mathrm{H})^{+} ; \mathrm{FAB}+\mathrm{m} / z 454(\mathrm{M}+\mathrm{H})^{+}, 476(\mathrm{M}+\mathrm{Na})^{+}$; HRMS calcd for $\mathrm{C}_{21} \mathrm{H}_{32} \mathrm{NO}_{6} \mathrm{SSi} 454.1720$, found 454.1720.

Methyl 2-((N-Allyl-2-(trimethylsilyl)ethan-3-ylsulfonamido)(3-fluorophenyl)methyl)acrylate (6e). Alkylation of the $\beta$-aminoester 5e yielded $102 \mathrm{mg}(99 \%)$ of the title compound as a pale yellow oil.

IR $2893(\mathrm{~m}), 1725(\mathrm{~s}), 1331(\mathrm{~s}) \mathrm{cm}^{-1} ;{ }^{1} \mathrm{H} \mathrm{NMR}\left(\mathrm{CDCl}_{3}, \mathrm{Me}_{4-}\right.$ Si) $\delta 0.05(\mathrm{~s}, 9 \mathrm{H}), 0.95-1.15(\mathrm{~m}, 2 \mathrm{H}), 2.80-3.10(\mathrm{~m}, 2 \mathrm{H}), 3.74$ $(\mathrm{s}, 3 \mathrm{H}), 3.80-4.10(\mathrm{~m}, 2 \mathrm{H}), 4.95-5.15(\mathrm{~m}, 2 \mathrm{H}), 5.25-5.55(\mathrm{~m}$, $1 \mathrm{H}), 5.88\left(\mathrm{~d}, 1 \mathrm{H}, J_{4}=1.7 \mathrm{~Hz}\right), 6.09(\mathrm{~s}, 1 \mathrm{H}), 6.60\left(\mathrm{~d}, 1 \mathrm{H}, J_{4}=\right.$ $1.1 \mathrm{~Hz}), 6.95-7.15(\mathrm{~m}, 3 \mathrm{H}), 7.25-7.45(\mathrm{~m}, 1 \mathrm{H}) ;{ }^{13} \mathrm{C} \mathrm{NMR}$ $\left(\mathrm{CDCl}_{3}, \mathrm{Me}_{4} \mathrm{Si}\right) \delta-1.6,10.7,49.1,51.2,52.7,61.7\left(\mathrm{~d}, J_{4}=1.4\right.$ $\mathrm{Hz}), 115.5\left(\mathrm{~d}, J_{2}=21.2 \mathrm{~Hz}\right), 116.0\left(\mathrm{~d}, J_{2}=22.2 \mathrm{~Hz}\right), 119.2$, $124.5\left(\mathrm{~d}, J_{4}=2.8 \mathrm{~Hz}\right), 129.6,130.6\left(\mathrm{~d}, J_{3}=8.5 \mathrm{~Hz}\right), 134.5$, $139.8,141.0\left(\mathrm{~d}, J_{3}=6.7 \mathrm{~Hz}\right), 163.3\left(\mathrm{~d}, J_{1}=246.9 \mathrm{~Hz}\right), 166.6$; ESIMS $m / z 414.2(\mathrm{M}+\mathrm{H})^{+}, 436.2(\mathrm{M}+\mathrm{Na})^{+} ; \mathrm{FAB}+\mathrm{m} / z 414$ $(\mathrm{M}+\mathrm{H})^{+}, 436(\mathrm{M}+\mathrm{Na})^{+}$; HRMS calcd for $\mathrm{C}_{19} \mathrm{H}_{29} \mathrm{FNO}_{4} \mathrm{SSi}$ 414.1571, found 414.1620.

Methyl 2-( $(N$-Allyl-2-(trimethylsilyl)ethan-3-ylsulfonamido)(2-bromophenyl)methyl)acrylate (6f). Alkylation of the $\beta$-aminoester $5 \mathbf{f}$ yielded $117 \mathrm{mg}(99 \%)$ of the title compound as a pale yellow oil.

IR $2950(\mathrm{~m}), 1716(\mathrm{~s}), 1333(\mathrm{~s}) \mathrm{cm}^{-1} ;{ }^{1} \mathrm{H} \mathrm{NMR}\left(\mathrm{CDCl}_{3}, \mathrm{Me}_{4-}\right.$ Si) $\delta 0.03(\mathrm{~s}, 9 \mathrm{H}), 0.95-1.15(\mathrm{~m}, 2 \mathrm{H}), 2.80-3.05(\mathrm{~m}, 2 \mathrm{H}), 3.72$ $(\mathrm{s}, 3 \mathrm{H}), 4.08\left(\mathrm{dd}, 2 \mathrm{H}, J_{3}=6.5 \mathrm{~Hz}, J_{4}=1.1 \mathrm{~Hz}\right), 5.00-5.20(\mathrm{~m}$, $2 \mathrm{H}), 5.35-5.60(\mathrm{~m}, 1 \mathrm{H}), 5.88\left(\mathrm{~d}, 1 \mathrm{H}, J_{4}=1.5 \mathrm{~Hz}\right), 6.21(\mathrm{~d}, 1 \mathrm{H}$ $\left.J_{4}=0.8 \mathrm{~Hz}\right), 6.60(\mathrm{~d}, 1 \mathrm{H},=0.8 \mathrm{~Hz}), 7.20\left(\mathrm{ddd}, 1 \mathrm{H}, J_{4}=2.0\right.$ $\left.\mathrm{Hz}, J_{3}=7.2 \mathrm{~Hz}, J_{3}=7.8 \mathrm{~Hz}\right), 7.33\left(\mathrm{ddd}, 1 \mathrm{H}, J_{4}=1.3 \mathrm{~Hz}, J_{3}\right.$ $\left.=7.2 \mathrm{~Hz}, J_{3}=7.8 \mathrm{~Hz}\right), 7.42\left(\mathrm{dd}, 1 \mathrm{H}, J_{4}=2.0 \mathrm{~Hz}, J_{3}=7.8\right.$ $\mathrm{Hz}), 7.61\left(\mathrm{dd}, 1 \mathrm{H}, J_{4}=1.3 \mathrm{~Hz}, J_{3}=7.8 \mathrm{~Hz}\right) ;{ }^{13} \mathrm{C} \mathrm{NMR}\left(\mathrm{CDCl}_{3}\right.$, $\left.\mathrm{Me}_{4} \mathrm{Si}\right) \delta-1.6,10.5,50.5,51.4,52.6,62.6,119.0,125.0,128.0$, $129.8,130.0,130.8,133.8,134.7,137.9,139.4,166.4$; ESIMS $m / z 474.4 / 476.4(\mathrm{M}+\mathrm{H})^{+} ; \mathrm{FAB}+m / z 474 / 476(\mathrm{M}+\mathrm{H})^{+}$; HRMS calcd for $\mathrm{C}_{19} \mathrm{H}_{29} \mathrm{BrNO}_{4} \mathrm{SSi} 474.0770$, found 474.0749 .

Methyl 2-( $(N$-Allyl-2-(trimethylsilyl)ethan-3-ylsulfonamido)(naphthalen-2-yl)methyl)acrylate (6g). Alkylation of the $\beta$-aminoester $\mathbf{5 g}$ yielded $109 \mathrm{mg}(98 \%)$ of the title compound as a pale yellow oil.

IR $2953(\mathrm{~m}), 1723(\mathrm{~s}), 1333(\mathrm{~s}) \mathrm{cm}^{-1} ;{ }^{1} \mathrm{H} \mathrm{NMR}\left(\mathrm{CDCl}_{3}, \mathrm{Me}_{4}{ }^{-}\right.$ Si) $\delta 0.03(\mathrm{~s}, 9 \mathrm{H}), 1.00-1.15(\mathrm{~m}, 2 \mathrm{H}), 2.80-3.10(\mathrm{~m}, 2 \mathrm{H}), 3.71$ $(\mathrm{s}, 3 \mathrm{H}), 4.00\left(\mathrm{dd}, 2 \mathrm{H}, J_{4}=5.8 \mathrm{~Hz}, J_{3}=5.9 \mathrm{~Hz}\right), 4.90-5.15(\mathrm{~m}$, $2 \mathrm{H}), 5.20-5.45(\mathrm{~m}, 1 \mathrm{H}), 5.95\left(\mathrm{~d}, 1 \mathrm{H}, J_{4}=1.1 \mathrm{~Hz}\right), 6.27(\mathrm{~s}, 1 \mathrm{H})$, $6.64(\mathrm{~s}, 1 \mathrm{H}), 7.40-7.60(\mathrm{~m}, 3 \mathrm{H}), 7.70-7.90(\mathrm{~m}, 4 \mathrm{H}) ;{ }^{13} \mathrm{C} \mathrm{NMR}$ $\left(\mathrm{CDCl}_{3}, \mathrm{Me}_{4} \mathrm{Si}\right) \delta-1.6,10.7,49.0,51.2,52.6,62.5,119.1,126.9$, $127.0,127.9,128.0,128.6,128.9,129.0,133.3,133.6,134.7$, 135.6, 140.3, 166.9; ESIMS $m / z 446.4(\mathrm{M}+\mathrm{H})^{+}, 468.4(\mathrm{M}+$ $\mathrm{Na})^{+} ; \mathrm{FAB}+m / z 446(\mathrm{M}+\mathrm{H})^{+}, 468(\mathrm{M}+\mathrm{Na})^{+}$; HRMS calcd for $\mathrm{C}_{23} \mathrm{H}_{32} \mathrm{NO}_{4} \mathrm{SSi} 446.1821$, found 446.1814 .

Methyl 2-((N-Allyl-2-(trimethylsilyl)ethan-3-ylsulfonamido)(m-tolyl)methyl)acrylate (6h). Alkylation of the $\beta$-aminoester $\mathbf{5 h}$ yielded $100 \mathrm{mg}(98 \%)$ of the title compound as a pale yellow oil.

IR $2953(\mathrm{~m}), 1719(\mathrm{~s}), 1325(\mathrm{~s}) \mathrm{cm}^{-1} ;{ }^{1} \mathrm{H} \mathrm{NMR}\left(\mathrm{CDCl}_{3}, \mathrm{Me}_{4-}\right.$ Si) $\delta 0.04(\mathrm{~s}, 9 \mathrm{H}), 0.95-1.15(\mathrm{~m}, 2 \mathrm{H}), 2.37(\mathrm{~s}, 3 \mathrm{H}), 2.75-3.05$ $(\mathrm{m}, 2 \mathrm{H}), 3.70(\mathrm{~s}, 3 \mathrm{H}), 3.92\left(\mathrm{~d}, 2 \mathrm{H}, J_{3}=6.4 \mathrm{~Hz}\right), 4.95-5.15(\mathrm{~m}$, $2 \mathrm{H}), 5.15-5.40(\mathrm{~m}, 1 \mathrm{H}), 5.90\left(\mathrm{dd}, 1 \mathrm{H}, J_{2}=0.4 \mathrm{~Hz}, J_{4}=1.3\right.$ $\mathrm{Hz}), 6.05(\mathrm{~s}, 1 \mathrm{H}), 6.56\left(\mathrm{dd}, 1 \mathrm{H}, J_{2}=0.4 \mathrm{~Hz}, J_{4}=1.3 \mathrm{~Hz}\right), 7.05-$ $7.30(\mathrm{~m}, 4 \mathrm{H}) ;{ }^{13} \mathrm{C} \mathrm{NMR}\left(\mathrm{CDCl}_{3}, \mathrm{Me}_{4} \mathrm{Si}\right) \delta-1.6,10.7,21.9,49.0$ 51.0, 52.5, 62.4, 118.9, 126.0, 128.6, 129.0, 129.3, 129.8, 134.8, $137.9,138.8,140.3,166.9 ;$ ESIMS $m / z 410.2(\mathrm{M}+\mathrm{H})^{+}, 432.2$ $(\mathrm{M}+\mathrm{Na})^{+}, 841.3(2 \mathrm{M}+\mathrm{Na})^{+} ; \mathrm{FAB}+m / z 410(\mathrm{M}+\mathrm{H})^{+}, 432$ $(\mathrm{M}+\mathrm{Na})^{+}$; HRMS calcd for $\mathrm{C}_{20} \mathrm{H}_{32} \mathrm{NO}_{4} \mathrm{SSi}$ 410.1821, found 410.1841 . 
Methyl 2-( $(N$-Allyl-2-(trimethylsilyl)ethan-3-ylsulfonamido)(3,5-dimethylphenyl)methyl)acrylate (6i). Alkylation of the $\beta$-aminoester 5i yielded $103 \mathrm{mg}$ (97\%) of the title compound as a pale yellow oil.

IR $2953(\mathrm{~m}), 1721$ (s), 1331 (s) cm ${ }^{-1} ;{ }^{1} \mathrm{H} \mathrm{NMR}\left(\mathrm{CDCl}_{3}, \mathrm{Me}_{4^{-}}\right.$ Si) $\delta 0.04(\mathrm{~s}, 9 \mathrm{H}), 0.95-1.15(\mathrm{~m}, 2 \mathrm{H}), 2.32(\mathrm{~s}, 6 \mathrm{H}), 2.75-3.05$ $(\mathrm{m}, 2 \mathrm{H}), 3.70(\mathrm{~s}, 3 \mathrm{H}), 3.91\left(\mathrm{~d}, 2 \mathrm{H}, J_{3}=6.1 \mathrm{~Hz}\right), 4.95-5.15(\mathrm{~m}$, $2 \mathrm{H}), 5.15-5.40(\mathrm{~m}, 1 \mathrm{H}), 5.90\left(\mathrm{dd}, 1 \mathrm{H}, J_{2}=0.6 \mathrm{~Hz}, J_{4}=1.7\right.$ $\mathrm{Hz}), 6.01(\mathrm{~s}, 1 \mathrm{H}), 6.54\left(\mathrm{dd}, 1 \mathrm{H}, J_{2}=0.5 \mathrm{~Hz}, J_{4}=1.3 \mathrm{~Hz}\right), 6.91$ $(\mathrm{s}, 2 \mathrm{H}), 6.95(\mathrm{~s}, 1 \mathrm{H}) ;{ }^{13} \mathrm{C} \mathrm{NMR}\left(\mathrm{CDCl}_{3}, \mathrm{Me}_{4} \mathrm{Si}\right) \delta-1.6,10.7$, 21.8, 48.9, 51.0, 52.5, 62.4, 118.8, 126.8, 128.4, 130.2, 134.9, 137.8, 138.6, 140.5, 166.9; ESIMS $\mathrm{m} / z$ 424.3 $(\mathrm{M}+\mathrm{H})^{+} ; \mathrm{FAB}+$ $\mathrm{m} / z 424(\mathrm{M}+\mathrm{H})^{+}, 446(\mathrm{M}+\mathrm{Na})^{+}$; HRMS calcd for $\mathrm{C}_{21} \mathrm{H}_{34^{-}}$ $\mathrm{NO}_{4} \mathrm{SSi}$ 424.1978, found 424.2021.

Methyl 2-( $(N$-Allyl-2-(trimethylsilyl)ethan-3-ylsulfonamido)(2,3-methylenedioxyphenyl)methyl)acrylate (6j). Alkylation of the $\beta$-aminoester $\mathbf{5 j}$ yielded $108 \mathrm{mg}(98 \%)$ of the title compound as a pale yellow oil.

IR $2938(\mathrm{~m}), 1720(\mathrm{~s}), 1329(\mathrm{~s}) \mathrm{cm}^{-1} ;{ }^{1} \mathrm{H} \mathrm{NMR}\left(\mathrm{CDCl}_{3}, \mathrm{Me}_{4-}\right.$ Si) $\delta 0.05(\mathrm{~s}, 9 \mathrm{H}), 0.95-1.20(\mathrm{~m}, 2 \mathrm{H}), 2.80-3.15(\mathrm{~m}, 2 \mathrm{H}), 3.70$ (s, $3 \mathrm{H}), 3.89$ (dd, $1 \mathrm{H}, J_{2}=5.8 \mathrm{~Hz}, J_{3}=16.0 \mathrm{~Hz}$ ), 4.05 (dd, $1 \mathrm{H}$, $\left.J_{2}=7.2 \mathrm{~Hz}, J_{3}=16.0 \mathrm{~Hz}\right), 4.95-5.15(\mathrm{~m}, 2 \mathrm{H}), 5.20-5.45(\mathrm{~m}$, $1 \mathrm{H}), 5.95-6.00(\mathrm{~m}, 3 \mathrm{H}), 6.10(\mathrm{~s}, 1), 6.55\left(\mathrm{~d}, 1 \mathrm{H}, J_{4}=1.5 \mathrm{~Hz}\right)$, 6.70-6.90 (m, 3H); ${ }^{13} \mathrm{C} \mathrm{NMR}\left(\mathrm{CDCl}_{3}, \mathrm{Me}_{4} \mathrm{Si}\right) \delta-1.6,10.4,49.0$, $50.8,52.5,57.4,101.3,109.2,118.9,120.1,121.9,122.4,128.3$, 134.6, 139.1, 145.8, 147.8, 166.5; ESIMS $m / z$ 440.1 $(\mathrm{M}+\mathrm{H})^{+}$, $462.4(\mathrm{M}+\mathrm{Na})^{+} ; \mathrm{FAB}+\mathrm{m} / z 440(\mathrm{M}+\mathrm{H})^{+}, 444(\mathrm{M}+\mathrm{Na})^{+}$; HRMS calcd for $\mathrm{C}_{20} \mathrm{H}_{30} \mathrm{NO}_{6} \mathrm{SSi} 440.1563$, found 440.1534 .

Methyl 2-( $(\boldsymbol{N}$-Allyl-2-(trimethylsilyl)ethan-3-ylsulfonamido)(4-chlorophenyl)methyl)acrylate (6k). Alkylation of the $\beta$-aminoester 5k yielded $106 \mathbf{m g}$ (99\%) of the title compound as a pale yellow oil.

IR $2948(\mathrm{~m}), 1718(\mathrm{~s}), 1329(\mathrm{~s}) \mathrm{cm}^{-1} ;{ }^{1} \mathrm{H} \mathrm{NMR}\left(\mathrm{CDCl}_{3}, \mathrm{Me}_{4^{-}}\right.$ Si) $\delta 0.05(\mathrm{~s}, 9 \mathrm{H}), 0.95-1.10(\mathrm{~m}, 2 \mathrm{H}), 2.75-3.05(\mathrm{~m}, 2 \mathrm{H}), 3.73$ $(\mathrm{s}, 3 \mathrm{H}), 3.80-4.05(\mathrm{~m}, 2 \mathrm{H}), 5.00-5.15(\mathrm{~m}, 2 \mathrm{H}), 5.25-5.55(\mathrm{~m}$, $1 \mathrm{H}), 5.88\left(\mathrm{~d}, 1 \mathrm{H}, J_{4}=1.5 \mathrm{~Hz}\right), 6.05(\mathrm{~s}, 1 \mathrm{H}), 6.59\left(\mathrm{~d}, 1 \mathrm{H}, J_{4}=\right.$ $0.9 \mathrm{~Hz}), 7.25\left(\mathrm{~d}, 2 \mathrm{H}, J_{3}=8.4 \mathrm{~Hz}\right), 7.35\left(\mathrm{~d}, 2 \mathrm{H}, J_{3}=8.7 \mathrm{~Hz}\right)$; ${ }^{13} \mathrm{C} \mathrm{NMR}\left(\mathrm{CDCl}_{3}, \mathrm{Me}_{4} \mathrm{Si}\right) \delta-1.6,10.7,49.1,51.2,52.7,61.7$, 119.3, 129.3, 129.4, 130.3, 134.4, 134.8, 136.8, 139.8, 166.7; ESIMS $m / z 430.2(\mathrm{M}+\mathrm{H})^{+}, 452.0(\mathrm{M}+\mathrm{Na})^{+} ; \mathrm{FAB}+\mathrm{m} / z 430$ $(\mathrm{M}+\mathrm{H})^{+}, 452(\mathrm{M}+\mathrm{Na})^{+}$; HRMS calcd for $\mathrm{C}_{19} \mathrm{H}_{28} \mathrm{ClNO}_{4} \mathrm{SSi}$ 430.1275 , found 430.1266

Methyl 2-( $(N$-Allyl-2-(trimethylsilyl)ethan-3-ylsulfonamido)(2-iodophenyl)methyl)acrylate (6l). Alkylation of the $\beta$-aminoester $\boldsymbol{5} \boldsymbol{l}$ yielded $129 \mathrm{mg}$ (99\%) of the title compound as a pale yellow oil.

IR $2950(\mathrm{~m}), 1723(\mathrm{~s}), 1331(\mathrm{~s}) \mathrm{cm}^{-1} ;{ }^{1} \mathrm{H} \mathrm{NMR}\left(\mathrm{CDCl}_{3}, \mathrm{Me}_{4^{-}}\right.$ Si) $\delta 0.04(\mathrm{~s}, 9 \mathrm{H}), 1.00-1.15(\mathrm{~m}, 2 \mathrm{H}), 2.90-3.05(\mathrm{~m}, 2 \mathrm{H}), 3.74$ $(\mathrm{s}, 3 \mathrm{H}), 4.10\left(\mathrm{~d}, 2 \mathrm{H}, J_{3}=6.7 \mathrm{~Hz}\right), 5.00-5.20(\mathrm{~m}, 2 \mathrm{H}), 5.35-$ $5.60(\mathrm{~m}, 1 \mathrm{H}), 5.83\left(\mathrm{~d}, 1 \mathrm{H}, J_{4}=1.5 \mathrm{~Hz}\right), 6.08(\mathrm{~s}, 1 \mathrm{H}), 6.61(\mathrm{~d}$, $\left.1 \mathrm{H}, J_{4}=0.7 \mathrm{~Hz}\right), 6.95-7.10(\mathrm{~m}, 1 \mathrm{H}), 7.30-7.45(\mathrm{~m}, 2 \mathrm{H}), 7.85-$ $7.95(\mathrm{~m}, 1 \mathrm{H}) ;{ }^{13} \mathrm{C} \mathrm{NMR}\left(\mathrm{CDCl}_{3}, \mathrm{Me}_{4} \mathrm{Si}\right) \delta-1.5,10.5,50.6,51.5$, $52.6,67.1,101.2,118.9,128.8,130.1,130.3,130.3,134.8,139.5$, 140.7, 141.2, 166.4; ESIMS $\mathrm{m} / z 522.3(\mathrm{M}+\mathrm{H})^{+}, 544.4(\mathrm{M}+$ $\mathrm{Na})^{+} ; \mathrm{FAB}+m / z 522(\mathrm{M}+\mathrm{H})^{+}, 544(\mathrm{M}+\mathrm{Na})^{+} ; \mathrm{HRMS}$ calcd for $\mathrm{C}_{19} \mathrm{H}_{29} \mathrm{INO}_{4} \mathrm{SSi} 522.0631$, found 522.0655 .

Methyl 2-( $(\boldsymbol{N}$-Allyl-2-(trimethylsilyl)ethan-3-ylsulfonamido)(4-(2-(trimethylsilyl)ethynyl)phenyl)methyl)acrylate (6m). Alkylation of the $\beta$-aminoester $\mathbf{5 m}$ yielded 120 $\mathrm{mg}(98 \%)$ of the title compound as a pale yellow oil.

IR $2953(\mathrm{~m}), 1723(\mathrm{~s}), 1334(\mathrm{~s}) \mathrm{cm}^{-1} ;{ }^{1} \mathrm{H} \mathrm{NMR}\left(\mathrm{CDCl}_{3}, \mathrm{Me}_{4^{-}}\right.$ Si) $\delta 0.05(\mathrm{~s}, 9 \mathrm{H}), 0.27(\mathrm{~s}, 9 \mathrm{H}), 0.95-1.10(\mathrm{~m}, 2 \mathrm{H}), 2.80-3.05$ $(\mathrm{m}, 2 \mathrm{H}), 3.69(\mathrm{~s}, 3 \mathrm{H}), 3.90\left(\mathrm{~d}, 2 \mathrm{H}, J_{3}=6.3 \mathrm{~Hz}\right), 4.95-5.15(\mathrm{~m}$, $2 \mathrm{H}), 5.20-5.45(\mathrm{~m}, 1 \mathrm{H}), 5.87\left(\mathrm{~d}, 1 \mathrm{H}, J_{4}=1.5 \mathrm{~Hz}\right), 6.06(\mathrm{~s}, 1 \mathrm{H})$, $6.58\left(\mathrm{~d}, 1 \mathrm{H}, J_{4}=1.1 \mathrm{~Hz}\right), 7.25\left(\mathrm{~d}, 1 \mathrm{H}, J_{3}=8.4 \mathrm{~Hz}\right), 7.47(\mathrm{~d}$, $\left.1 \mathrm{H}, J_{3}=8.4 \mathrm{~Hz}\right) ;{ }^{13} \mathrm{C} \mathrm{NMR}\left(\mathrm{CDCl}_{3}, \mathrm{Me}_{4} \mathrm{Si}\right) \delta-2.0,-0.1,10.3$, 48.6, 50.8, 52.2, 61.8, 95.1, 104.4, 118.8, 123.0, 128.4, 128.7, 132.3, 134.1, 138.2, 139.5, 166.3; ESIMS $m / z$ 492.4 $(\mathrm{M}+\mathrm{H})^{+}$; $\mathrm{FAB}+\mathrm{m} / z 492(\mathrm{M}+\mathrm{H})^{+}, 514(\mathrm{M}+\mathrm{Na})^{+}$; HRMS calcd for $\mathrm{C}_{24} \mathrm{H}_{38} \mathrm{NO}_{4} \mathrm{SSi}_{2}$ 492.2060, found 492.2047.

Methyl 2-( $(N$-Allyl-2-(trimethylsilyl)ethan-3-ylsulfonamido)(4-nitrophenyl)methyl)acrylate (6n). Alkylation of the $\beta$-aminoester $5 n$ yielded $107 \mathrm{mg}$ (97\%) of the title compound as a pale yellow oil.

IR $2950(\mathrm{~m}), 1723$ (s), $1528(\mathrm{~s}), 1350$ (s), $1335(\mathrm{~s}) \mathrm{cm}^{-1} ;{ }^{1} \mathrm{H}$ $\operatorname{NMR}\left(\mathrm{CDCl}_{3}, \mathrm{Me}_{4} \mathrm{Si}\right) \delta 0.06(\mathrm{~s}, 9 \mathrm{H}), 0.90-1.10(\mathrm{~m}, 2 \mathrm{H}), 2.85-$ $3.10(\mathrm{~m}, 2 \mathrm{H}), 3.79(\mathrm{~s}, 3 \mathrm{H}), 3.93\left(\mathrm{~d}, 1 \mathrm{H}, J_{3}=7.0 \mathrm{~Hz}\right), 4.00(\mathrm{~d}$, $\left.1 \mathrm{H}, J_{3}=5.7 \mathrm{~Hz}\right), 5.00-5.20(\mathrm{~m}, 2 \mathrm{H}), 5.35-5.60(\mathrm{~m}, 1 \mathrm{H}), 5.80$ $\left(\mathrm{d}, 1 \mathrm{H}, J_{4}=1.5 \mathrm{~Hz}\right), 6.19(\mathrm{~s}, 1 \mathrm{H}), 6.67\left(\mathrm{~d}, 1 \mathrm{H}, J_{4}=0.9 \mathrm{~Hz}\right)$, $7.51\left(\mathrm{~d}, 2 \mathrm{H}, J_{3}=8.7 \mathrm{~Hz}\right), 8.23\left(\mathrm{~d}, 2 \mathrm{H}, J_{3}=8.7 \mathrm{~Hz}\right) ;{ }^{13} \mathrm{C} \mathrm{NMR}$ $\left(\mathrm{CDCl}_{3}, \mathrm{Me}_{4} \mathrm{Si}\right) \delta-1.6,10.6,49.3,51.3,52.9,61.6,119.7,124.2$, 129.6, 131.0, 134.2, 139.2, 146.3, 147.8, 166.5; ESIMS m/z $396.3(\mathrm{M}+\mathrm{H})^{+}, 791.7(2 \mathrm{M}+\mathrm{H})^{+} ; \mathrm{FAB}+\mathrm{m} / z 441(\mathrm{M}+\mathrm{H})^{+}$, $463(\mathrm{M}+\mathrm{Na})^{+}$; HRMS calcd for $\mathrm{C}_{19} \mathrm{H}_{29} \mathrm{~N}_{2} \mathrm{O}_{6} \mathrm{SSi}$ 441.1516, found 441.1517 .

General Procedure for the Synthesis of 2,5-Dihydropyrrole Derivatives 7: Thermal Conditions. $\mathrm{Cl}_{2}\left(\mathrm{PCy}_{3}\right)-$ $(\mathrm{IMes}) \mathrm{Ru}=\mathrm{CHPh}(8 \mathrm{mg}, 0.01 \mathrm{mmol})$ was added to a solution ${ }^{12 \mathrm{a}}$ of $N$-allyl- $\beta$-aminoester $6(0.20 \mathrm{mmol})$ in $20 \mathrm{~mL}$ of $\mathrm{CH}_{2} \mathrm{Cl}_{2}$, and the mixture was stirred overnight at room temperature. Then $35 \mu \mathrm{L}$ of DMSO (50 equiv/Ru) was added and the mixture was stirred for $24 \mathrm{~h}$. The solution was evaporated. Silica gel chromatography $\left(\mathrm{Et}_{2} \mathrm{O} /\right.$ hexane) yielded the corresponding 2,5dihydropyrrole 7 .

Microwave Irradiation. A solution ${ }^{14 a}$ of $\mathrm{Cl}_{2}\left(\mathrm{PCy}_{3}\right)(\mathrm{IMes})-$ $\mathrm{Ru}=\mathrm{CHPh}$ in $\mathrm{CH}_{2} \mathrm{Cl}_{2}(0.5 \mathrm{mM}, 0.5 \mathrm{~mL})$ was added to $N$-allyl$\beta$-aminoester $6(0.005 \mathrm{mmol})$, and the mixture was irradiated by microwave $5 \mathrm{~min}$ at $100{ }^{\circ} \mathrm{C}$. Filtration through a plug of silica yielded the corresponding 2,5-dihydropyrrole 7 .

Methyl 2-Phenyl-1-(2-(trimethylsilyl)ethylsulfonyl)2,5-dihydro-1H-pyrrole-3-carboxylate (7a): Thermal Conditions. The crude product was purified by silica gel chromatography $\left(\mathrm{Et}_{2} \mathrm{O} /\right.$ hexane $\left.=3 / 7\right)$ to yield $72 \mathrm{mg}(98 \%)$ of the title compound ${ }^{4 \mathrm{a}}$ as a white solid.

Microwave Irradiation. Cyclization of the compound $\mathbf{6 a}$ yielded $1.8 \mathrm{mg}(98 \%)$ of the title compound.

Mp 95.6-97.0 ${ }^{\circ} \mathrm{C}$ dec; IR 2950 (m), 1729 (s), 1340 (s), 1335 (s) $\mathrm{cm}^{-1} ;{ }^{1} \mathrm{H}$ NMR $\left(\mathrm{CDCl}_{3}, \mathrm{Me}_{4} \mathrm{Si}\right) \delta-0.15(\mathrm{~s}, 9 \mathrm{H}), 0.55-0.95$ $(\mathrm{m}, 2 \mathrm{H}), 2.20-2.50(\mathrm{~m}, 2 \mathrm{H}), 3.65(\mathrm{~s}, 3 \mathrm{H}), 4.41\left(\mathrm{ddd}, 1 \mathrm{H}, J_{2}=\right.$ $\left.17.1 \mathrm{~Hz}, J_{3}=6.2 \mathrm{~Hz}, J_{4}=2.0 \mathrm{~Hz}\right), 4.79\left(\mathrm{dt}, 1 \mathrm{H}, J_{2}=17.1 \mathrm{~Hz}\right.$, $\left.J_{3}=2.6 \mathrm{~Hz}\right), 5.84\left(\mathrm{ddd}, 1 \mathrm{H}, J_{4}=6.1 \mathrm{~Hz}, J_{4}=2.6 \mathrm{~Hz}, J_{4}=1.7\right.$ $\mathrm{Hz}), 6.95\left(\mathrm{dt}, 1 \mathrm{H}, J_{3}=2.2 \mathrm{~Hz}, J_{4}=1.8 \mathrm{~Hz}\right), 7.30-7.45(\mathrm{~m}$, $5 \mathrm{H}) ;{ }^{13} \mathrm{C} \mathrm{NMR}\left(\mathrm{CDCl}_{3}, \mathrm{Me}_{4} \mathrm{Si}\right) \delta-1.8,10.3,50.3,52.3,55.9$, $68.8,128.2,129.0,129.1,135.8,136.5,140.0,162.7$; ESIMS $\mathrm{m} / \mathrm{z} 368.0(\mathrm{M}+\mathrm{H})^{+}, 390.2(\mathrm{M}+\mathrm{Na})^{+}, 735.3(2 \mathrm{M}+\mathrm{H})^{+}, 757.1$ $(2 \mathrm{M}+\mathrm{Na})^{+} ; \mathrm{FAB}+m / z 368(\mathrm{M}+\mathrm{H})^{+} ; \mathrm{HRMS}$ calcd for $\mathrm{C}_{17} \mathrm{H}_{26^{-}}$ $\mathrm{NO}_{4} \mathrm{SSi} 368.1352$, found 368.1349 .

Methyl 2-(3,5-Dimethoxyphenyl)-1-(2-(trimethylsilyl)ethylsulfonyl)-2,5-dihydro- $1 H$-pyrrole-3-carboxylate (7b): Thermal Conditions. The crude product was purified by silica gel chromatography $\left(\mathrm{Et}_{2} \mathrm{O} /\right.$ hexane $\left.=4 / 6\right)$ to yield 82 $\mathrm{mg}(95 \%)$ of the title compound as a white solid.

Microwave Irradiation. Cyclization of the compound $\mathbf{6 b}$ yielded $2.0 \mathrm{mg}(92 \%)$ of the title compound.

Mp 101.0-105.0 ${ }^{\circ} \mathrm{C}$ dec; IR 2950 (m), 1729 (s), 1344 (s) cm ${ }^{-1}$; ${ }^{1} \mathrm{H}$ NMR $\left(\mathrm{CDCl}_{3}, \mathrm{Me}_{4} \mathrm{Si}\right) \delta-0.12(\mathrm{~s}, 9 \mathrm{H}), 0.60-1.00(\mathrm{~m}, 2 \mathrm{H})$, $2.25-2.60(\mathrm{~m}, 2 \mathrm{H}), 3.67(\mathrm{~s}, 3 \mathrm{H}), 3.80(\mathrm{~s}, 6 \mathrm{H}), 4.39$ (ddd, $1 \mathrm{H}, J_{2}$ $\left.=17.1 \mathrm{~Hz}, J_{3}=6.1 \mathrm{~Hz}, J_{4}=2.0 \mathrm{~Hz}\right), 4.77\left(\mathrm{dt}, 1 \mathrm{H}, J_{2}=17.1\right.$ $\left.\mathrm{Hz}, J_{3}=2.6 \mathrm{~Hz}\right), 5.76\left(\mathrm{ddd}, 1 \mathrm{H}, J_{4}=6.1 \mathrm{~Hz}, J_{4}=2.4 \mathrm{~Hz}, J_{4}\right.$ $=1.7 \mathrm{~Hz}), 6.41\left(\mathrm{t}, 1 \mathrm{H}, J_{3}=2.2 \mathrm{~Hz}\right), 6.51(\mathrm{~d}, 2 \mathrm{H}), 6.93(\mathrm{q}, 1 \mathrm{H}$ $\left.J_{3}=2.0 \mathrm{~Hz}, J_{4}=1.8 \mathrm{~Hz}\right) ;{ }^{13} \mathrm{C} \mathrm{NMR}\left(\mathrm{CDCl}_{3}, \mathrm{Me}_{4} \mathrm{Si}\right) \delta-1.9$, $10.5,50.4,52.3,55.7,56.0,68.7,100.4,106.4,135.6,136.6$, 142.3, 161.4, 162.7; ESIMS $m / z 428.1(\mathrm{M}+\mathrm{H})^{+}, 450.2(\mathrm{M}+$ $\mathrm{Na})^{+} ; \mathrm{FAB}+\mathrm{m} / z 427\left(\mathrm{M}+\mathrm{e}^{-}\right)^{+}, 428(\mathrm{M}+\mathrm{H})^{+}$; HRMS calcd for $\mathrm{C}_{19} \mathrm{H}_{30} \mathrm{NO}_{6} \mathrm{SSi} 428.1563$, found 428.1582 .

Methyl 2-(4-(Methoxycarbonyl)phenyl)-1-(2-(trimethylsilyl)ethylsulfonyl)-2,5-dihydro-1 $H$-pyrrole-3-carboxylate (7c): Thermal Conditions. The crude product was purified by silica gel chromatography $\left(\mathrm{Et}_{2} \mathrm{O} /\right.$ hexane $\left.=4 / 6\right)$ to yield $78 \mathrm{mg}(92 \%)$ of the title compound as a white solid.

Microwave Irradiation. Cyclization of the compound $\mathbf{6 c}$ yielded $2.0 \mathrm{mg}(92 \%)$ of the title compound.

Mp 106.4-107.4 ${ }^{\circ} \mathrm{C}$; IR 2950 (m), 1731 (s), 1348 (s) cm ${ }^{-1}$; ${ }^{1} \mathrm{H} \mathrm{NMR}\left(\mathrm{CDCl}_{3}, \mathrm{Me}_{4} \mathrm{Si}\right) \delta-0.15(\mathrm{~s}, 9 \mathrm{H}), 0.55-0.95(\mathrm{~m}, 2 \mathrm{H})$, 
$2.25-2.60(\mathrm{~m}, 2 \mathrm{H}), 3.65(\mathrm{~s}, 3 \mathrm{H}), 3.93(\mathrm{~s}, 3 \mathrm{H}), 4.44\left(\mathrm{ddd}, 1 \mathrm{H}, J_{2}\right.$ $\left.=17.1 \mathrm{~Hz}, J_{3}=6.1 \mathrm{~Hz}, J_{4}=1.9 \mathrm{~Hz}\right), 4.80\left(\mathrm{dt}, 1 \mathrm{H}, J_{2}=17.1\right.$ $\left.\mathrm{Hz}, J_{3}=2.6 \mathrm{~Hz}\right), 5.90\left(\mathrm{ddd}, 1 \mathrm{H}, J_{4}=6.1 \mathrm{~Hz}, J_{4}=2.6 \mathrm{~Hz}, J_{4}\right.$ $=1.9 \mathrm{~Hz}), 6.98\left(\mathrm{q}, 1 \mathrm{H}, J_{3}=2.0 \mathrm{~Hz}\right), 7.46\left(\mathrm{ddd}, 2 \mathrm{H}, J_{3}=8.5\right.$ $\left.\mathrm{Hz}, J_{4}=1.9 \mathrm{~Hz}\right), 8.06\left(\mathrm{ddd}, 2 \mathrm{H}, J_{3}=8.5 \mathrm{~Hz}, J_{4}=1.9 \mathrm{~Hz}\right) ;{ }^{13} \mathrm{C}$ $\mathrm{NMR}\left(\mathrm{CDCl}_{3}, \mathrm{Me}_{4} \mathrm{Si}\right) \delta-1.8,10.3,50.2,52.4,52.6,56.1,68.5$, 128.2, 130.4, 130.8, 135.4, 137.1, 145.2, 162.5, 166.9; ESIMS $\mathrm{m} / \mathrm{z} 426.2(\mathrm{M}+\mathrm{H})^{+}, 851.2(2 \mathrm{M}+\mathrm{H})^{+}, 873.3(2 \mathrm{M}+\mathrm{Na})^{+} ; \mathrm{FAB}+$ $m / z 426(\mathrm{M}+\mathrm{H})^{+}, 448(\mathrm{M}+\mathrm{Na})^{+}$; HRMS calcd for $\mathrm{C}_{19} \mathrm{H}_{28^{-}}$ $\mathrm{NO}_{6} \mathrm{SSi} 426.1407$, found 426.1403.

Methyl 2-(3-Fluorophenyl)-1-(2-(trimethylsilyl)ethylsulfonyl)-2,5-dihydro-1H-pyrrole-3-carboxylate (7e): Thermal Conditions. The crude product was purified by silica gel chromatography $\left(\mathrm{Et}_{2} \mathrm{O} /\right.$ hexane $\left.=3 / 7\right)$ to yield $73 \mathrm{mg}$ $(95 \%)$ of the title compound as a white solid.

Mp 80.1-80.7 ${ }^{\circ} \mathrm{C}$ dec; IR 2950 (m), 1731 (s), 1346 (s) cm ${ }^{-1}$; ${ }^{1} \mathrm{H}$ NMR $\left(\mathrm{CDCl}_{3}, \mathrm{Me}_{4} \mathrm{Si}\right) \delta-0.12(\mathrm{~s}, 9 \mathrm{H}), 0.60-0.95(\mathrm{~m}, 2 \mathrm{H})$, $2.25-2.60(\mathrm{~m}, 2 \mathrm{H}), 3.66(\mathrm{~s}, 3 \mathrm{H}), 4.42\left(\mathrm{ddd}, 1 \mathrm{H}, J_{2}=2.0 \mathrm{~Hz}, J_{3}\right.$ $\left.=6.1 \mathrm{~Hz}, J_{4}=17.1 \mathrm{~Hz}\right), 4.78\left(\mathrm{dt}, 1 \mathrm{H}, J_{2}=2.6 \mathrm{~Hz}, J_{4}=17.1\right.$ $\mathrm{Hz}), 5.85(\mathrm{~m}, 1 \mathrm{H}), 6.90-7.10(\mathrm{~m}, 3 \mathrm{H}), 7.15-7.25(\mathrm{~m}, 1 \mathrm{H}), 7.30-$ $7.45(\mathrm{~m}, 1 \mathrm{H}) ;{ }^{13} \mathrm{C} \mathrm{NMR}\left(\mathrm{CDCl}_{3}, \mathrm{Me}_{4} \mathrm{Si}\right) \delta-1.8,10.3,50.3,52.4$, $56.0,68.3\left(\mathrm{~d}, J_{4}=1.6 \mathrm{~Hz}\right), 114.9\left(\mathrm{~d}, J_{2}=22.0 \mathrm{~Hz}\right), 116.0\left(\mathrm{~d}, J_{2}\right.$ $=21.2 \mathrm{~Hz}), 124.1\left(\mathrm{~d}, J_{4}=2.8 \mathrm{~Hz}\right), 130.7\left(\mathrm{~d}, J_{3}=7.8 \mathrm{~Hz}\right), 135.4$, $136.9,142.8\left(\mathrm{~d}, J_{3}=6.1 \mathrm{~Hz}\right), 162.6,163.3\left(\mathrm{~d}, J_{1}=247.5 \mathrm{~Hz}\right)$; ESIMS $m / z 386.1(\mathrm{M}+\mathrm{H})^{+}, 771.2(2 \mathrm{M}+\mathrm{H})^{+}, 793.2(2 \mathrm{M}+$ $\mathrm{Na})^{+} ; \mathrm{FAB}+\mathrm{m} / \mathrm{z} 386(\mathrm{M}+\mathrm{H})^{+}, 408(\mathrm{M}+\mathrm{Na})^{+}, 771(2 \mathrm{M}+$ $\mathrm{H})^{+}, 793(2 \mathrm{M}+\mathrm{Na})^{+}$; HRMS calcd for $\mathrm{C}_{17} \mathrm{H}_{25} \mathrm{FNO}_{4} \mathrm{SSi} 386.1258$, found 386.1234 .

Methyl 2-(2-Bromophenyl)-1-(2-(trimethylsilyl)ethylsulfonyl)-2,5-dihydro-1H-pyrrole-3-carboxylate (7f): Thermal Conditions. The crude product was purified by silica gel chromatography $\left(\mathrm{Et}_{2} \mathrm{O} /\right.$ hexane $\left.=3 / 7\right)$ to yield $80 \mathrm{mg}(90 \%)$ of the title compound as a white solid.

Microwave Irradiation. Cyclization of compound $6 \mathbf{f}$ yielded $2.0 \mathrm{mg}(90 \%)$ of the title compound.

Mp 88.7-90.1 ${ }^{\circ} \mathrm{C}$ dec; IR 2950 (m), 1728 (s), 1340 (s) $\mathrm{cm}^{-1}$; ${ }^{1} \mathrm{H}$ NMR $\left(\mathrm{CDCl}_{3}, \mathrm{Me}_{4} \mathrm{Si}\right) \delta-0.11(\mathrm{~s}, 9 \mathrm{H}), 0.65-1.00(\mathrm{~m}, 2 \mathrm{H})$, $2.35-2.70(\mathrm{~m}, 2 \mathrm{H}), 3.66(\mathrm{~s}, 3 \mathrm{H}), 4.47\left(\mathrm{ddd}, 1 \mathrm{H}, J_{2}=17.1 \mathrm{~Hz}\right.$, $\left.J_{3}=6.3 \mathrm{~Hz}, J_{4}=2.0 \mathrm{~Hz}\right), 4.83\left(\mathrm{dt}, 1 \mathrm{H}, J_{2}=17.1 \mathrm{~Hz}, J_{3}=2.8\right.$ $\mathrm{Hz}), 6.31$ (ddd, $1 \mathrm{H}, J_{4}=6.3 \mathrm{~Hz}, J_{4}=2.8 \mathrm{~Hz}, J_{4}=2.0 \mathrm{~Hz}$ ), $6.95\left(\mathrm{q}, 1 \mathrm{H}, J_{3}=2.0 \mathrm{~Hz}\right), 7.10-7.40(\mathrm{~m}, 3 \mathrm{H}), 7.55-7.65(\mathrm{~m}$, $1 \mathrm{H}) ;{ }^{13} \mathrm{C} \mathrm{NMR}\left(\mathrm{CDCl}_{3}, \mathrm{Me}_{4} \mathrm{Si}\right) \delta-2.2,9.8,49.7,51.9,55.9,67.9$, 123.6, 127.8, 129.8, 130.4, 133.7, 134.7, 136.8, 138.7, 162.0; ESIMS $m / z 445.9 / 447.9(\mathrm{M}+\mathrm{H})^{+}$; FAB $+m / z 446 / 448(\mathrm{M}+$ $\mathrm{H})^{+}$; HRMS calcd for $\mathrm{C}_{17} \mathrm{H}_{25} \mathrm{BrNO}_{4} \mathrm{SSi}$ 446.0457, found 446.0427.

Methyl 2-(Naphthalen-2-yl)-1-(2-(trimethylsilyl)ethylsulfonyl)-2,5-dihydro-1H-pyrrole-3-carboxylate $(7 \mathrm{~g})$ : Thermal Conditions. The crude product was purified by silica gel chromatography $\left(\mathrm{Et}_{2} \mathrm{O} /\right.$ hexane $\left.=3 / 7\right)$ to yield $75 \mathrm{mg}(90 \%)$ of the title compound as a white solid.

Microwave Irradiation. Cyclization of compound $\mathbf{6 g}$ yielded $1.9 \mathrm{mg}(89 \%)$ of the title compound.

Mp 123.1-124.3 ${ }^{\circ} \mathrm{C}$; IR 2950 (m), 1728 (s), 1339 (s) cm ${ }^{-1}$; ${ }^{1} \mathrm{H}$ NMR $\left(\mathrm{CDCl}_{3}, \mathrm{Me}_{4} \mathrm{Si}\right) \delta-0.37(\mathrm{~s}, 9 \mathrm{H}), 0.50-0.85(\mathrm{~m}, 2 \mathrm{H})$, $2.10-2.45(\mathrm{~m}, 2 \mathrm{H}), 3.62(\mathrm{~s}, 3 \mathrm{H}), 4.49\left(\mathrm{ddd}, 1 \mathrm{H}, J_{2}=17.1 \mathrm{~Hz}\right.$, $\left.J_{3}=6.3 \mathrm{~Hz}, J_{4}=2.0 \mathrm{~Hz}\right), 4.84\left(\mathrm{dt}, 1 \mathrm{H}, J_{2}=17.1 \mathrm{~Hz}, J_{3}=2.6\right.$ $\mathrm{Hz}$ ), 6.02 (ddd, $1 \mathrm{H}, J_{4}=6.1 \mathrm{~Hz}, J_{4}=2.6 \mathrm{~Hz}, J_{4}=1.7 \mathrm{~Hz}$ ), $7.02\left(\mathrm{dt}, 1 \mathrm{H}, J_{3}=2.0 \mathrm{~Hz}, J_{4}=1.8 \mathrm{~Hz}\right), 7.35-7.60(\mathrm{~m}, 3 \mathrm{H})$, $7.75-7.95(\mathrm{~m}, 4 \mathrm{H}) ;{ }^{13} \mathrm{C} \mathrm{NMR}\left(\mathrm{CDCl}_{3}, \mathrm{Me}_{4} \mathrm{Si}\right) \delta-2.1,10.2,50.3$, 52.3, 56.0, 69.0, 125.1, 126.9, 127.9, 128.1, 128.6, 129.1, 133.6, 133.8, 135.7, 136.7, 137.1, 162.7; ESIMS $m / z 418.2(\mathrm{M}+\mathrm{H})^{+}$, $440.0(\mathrm{M}+\mathrm{Na})^{+} ; \mathrm{FAB}+\mathrm{m} / z 417\left(\mathrm{M}-\mathrm{e}^{-}\right)^{+}, 440(\mathrm{M}+\mathrm{Na})^{+}$; HRMS calcd for $\mathrm{C}_{21} \mathrm{H}_{27} \mathrm{NO}_{4} \mathrm{SSi} 417.1430$, found 417.1421.

Methyl 2-m-Tolyl-1-(2-(trimethylsilyl)ethylsulfonyl)2,5-dihydro-1H-pyrrole-3-carboxylate $(7 \mathrm{~h})$ : Thermal Conditions. The crude product was purified by silica gel chromatography $\left(\mathrm{Et}_{2} \mathrm{O} /\right.$ hexane $\left.=3 / 7\right)$ to yield $75 \mathrm{mg}(98 \%)$ of the title compound as a white solid.

Mp 85.0-87.2 ${ }^{\circ} \mathrm{C}$; IR $2945(\mathrm{~m}), 1731$ (s), $1344(\mathrm{~s}) \mathrm{cm}^{-1} ;{ }^{1} \mathrm{H}$ $\mathrm{NMR}\left(\mathrm{CDCl}_{3}, \mathrm{Me}_{4} \mathrm{Si}\right) \delta-0.15(\mathrm{~s}, 9 \mathrm{H}), 0.55-0.95$ (m, 2H), 2.20$2.50(\mathrm{~m}, 2 \mathrm{H}), 2.37(\mathrm{~s}, 3 \mathrm{H}), 3.66(\mathrm{~s}, 3 \mathrm{H}), 4.41\left(\mathrm{ddd}, 1 \mathrm{H}, J_{2}=\right.$ $\left.17.1 \mathrm{~Hz}, J_{3}=6.3 \mathrm{~Hz}, J_{4}=2.0 \mathrm{~Hz}\right), 4.78\left(\mathrm{dt}, 1 \mathrm{H}, J_{2}=17.1 \mathrm{~Hz}\right.$,
$\left.J_{3}=2.6 \mathrm{~Hz}\right), 5.79\left(\mathrm{ddd}, 1 \mathrm{H}, J_{4}=6.1 \mathrm{~Hz}, J_{4}=2.6 \mathrm{~Hz}, J_{4}=1.7\right.$ $\mathrm{Hz}), 6.94\left(\mathrm{dt}, 1 \mathrm{H}, J_{3}=2.2 \mathrm{~Hz}, J_{4}=1.9 \mathrm{~Hz}\right), 7.05-7.35(\mathrm{~m}$, $4 \mathrm{H}) ;{ }^{13} \mathrm{C} \mathrm{NMR}\left(\mathrm{CDCl}_{3}, \mathrm{Me}_{4} \mathrm{Si}\right) \delta-1.8,10.3,21.8,50.2,52.3$, 55.9, 68.8, 125.3, 128.8, 129.0, 129.8, 135.8, 136.9, 138.7, 139.9, 162.76; ESIMS $m / z 382.1(\mathrm{M}+\mathrm{H})^{+}, 404.1(\mathrm{M}+\mathrm{Na})^{+}, 763.4$ $(2 \mathrm{M}+\mathrm{H})^{+} ; \mathrm{FAB}+\mathrm{m} / \mathrm{z} 382(\mathrm{M}+\mathrm{H})^{+}, 404(\mathrm{M}+\mathrm{Na})^{+} ;$HRMS calcd for $\mathrm{C}_{18} \mathrm{H}_{28} \mathrm{NO}_{4} \mathrm{SSi} 382.1508$, found 382.1505 .

Methyl 2-(3,5-Dimethylphenyl)-1-(2-(trimethylsilyl)ethylsulfonyl)-2,5-dihydro-1H-pyrrole-3-carboxylate (7i): Thermal Conditions. The crude product was purified by silica gel chromatography $\left(\mathrm{Et}_{2} \mathrm{O} /\right.$ hexane $\left.=3 / 7\right)$ to yield 73 $\mathrm{mg}(92 \%)$ of the title compound as a white solid.

Mp 96.0-97.2 ${ }^{\circ} \mathrm{C}$; IR $2950(\mathrm{~m}), 1731$ (s), $1344(\mathrm{~s}) \mathrm{cm}^{-1} ;{ }^{1} \mathrm{H}$ $\operatorname{NMR}\left(\mathrm{CDCl}_{3}, \mathrm{Me}_{4} \mathrm{Si}\right) \delta-0.15(\mathrm{~s}, 9 \mathrm{H}), 0.55-0.95(\mathrm{~m}, 2 \mathrm{H}), 2.20-$ $2.50(\mathrm{~m}, 2 \mathrm{H}), 2.32(\mathrm{~s}, 6 \mathrm{H}), 3.67(\mathrm{~s}, 3 \mathrm{H}), 4.40\left(\mathrm{ddd}, 1 \mathrm{H}, J_{2}=\right.$ $\left.17.1 \mathrm{~Hz}, J_{3}=6.1 \mathrm{~Hz}, J_{4}=2.0 \mathrm{~Hz}\right), 4.77\left(\mathrm{dt}, 1 \mathrm{H}, J_{2}=17.1 \mathrm{~Hz}\right.$, $\left.J_{3}=2.6 \mathrm{~Hz}\right), 5.75\left(\mathrm{ddd}, 1 \mathrm{H}, J_{4}=6.1 \mathrm{~Hz}, J_{4}=2.4 \mathrm{~Hz}, J_{4}=1.7\right.$ $\mathrm{Hz}), 6.90-7.00(\mathrm{~m}, 4 \mathrm{H}) ;{ }^{13} \mathrm{C} \mathrm{NMR}\left(\mathrm{CDCl}_{3}, \mathrm{Me}_{4} \mathrm{Si}\right) \delta-1.9,10.2$, 21.7, 50.2, 52.3, 55.8, 68.7, 125.9, 130.7, 135.9, 136.3, 138.6, 139.8, 162.8; ESIMS $\mathrm{m} / \mathrm{z} 396.1(\mathrm{M}+\mathrm{H})^{+}, 418.1(\mathrm{M}+\mathrm{Na})^{+}$; $791.4(2 \mathrm{M}+\mathrm{H})^{+}, 813.2(2 \mathrm{M}+\mathrm{Na})^{+} ; \mathrm{FAB}+m / z 396(\mathrm{M}+\mathrm{H})^{+}$, $418(\mathrm{M}+\mathrm{Na})^{+} ;$HRMS calcd for $\mathrm{C}_{19} \mathrm{H}_{30} \mathrm{NO}_{4} \mathrm{SSi} 396.1665$, found 396.1651 .

Methyl 2-(2,3-Methylenedioxyphenyl)-1-(2-(trimethylsilyl)ethylsulfonyl)-2,5-dihydro- $1 \mathrm{H}$-pyrrole-3-carboxylate (7j): Thermal Conditions. The crude product was purified by silica gel chromatography $\left(\mathrm{Et}_{2} \mathrm{O} /\right.$ hexane $\left.=4 / 6\right)$ to yield $79 \mathrm{mg}(96 \%)$ of the title compound as a white solid.

Mp 123.5-124.6 ${ }^{\circ} \mathrm{C} \mathrm{dec}$; IR $2953(\mathrm{~m}), 1729(\mathrm{~s}), 1356(\mathrm{~s}) \mathrm{cm}^{-1}$; ${ }^{1} \mathrm{H}$ NMR $\left(\mathrm{CDCl}_{3}, \mathrm{Me}_{4} \mathrm{Si}\right) \delta-0.10(\mathrm{~s}, 9 \mathrm{H}), 0.65-1.00(\mathrm{~m}, 2 \mathrm{H})$, $2.40-2.70(\mathrm{~m}, 2 \mathrm{H}), 3.68(\mathrm{~s}, 3 \mathrm{H}), 4.44\left(\mathrm{ddd}, 1 \mathrm{H}, J_{2}=16.7 \mathrm{~Hz}\right.$, $\left.J_{3}=6.1 \mathrm{~Hz}, J_{4}=1.9 \mathrm{~Hz}\right), 4.76\left(\mathrm{dt}, 1 \mathrm{H}, J_{2}=16.7 \mathrm{~Hz}, J_{3}=2.4\right.$ $\mathrm{Hz}), 5.84\left(\mathrm{ddd}, 1 \mathrm{H}, J_{4}=6.1 \mathrm{~Hz}, J_{4}=2.4 \mathrm{~Hz}, J_{4}=1.7 \mathrm{~Hz}\right.$ ), $5.98(\mathrm{~s}, 2 \mathrm{H}), 6.75-6.95(\mathrm{~m}, 4 \mathrm{H}) ;{ }^{13} \mathrm{C} \mathrm{NMR}\left(\mathrm{CDCl}_{3}, \mathrm{Me}_{4} \mathrm{Si}\right) \delta$ $-1.8,10.2,50.0,52.3,56.0,64.7,101.5,109.1,121.2,122.2$, 122.4, 133.8, 137.3, 145.6, 148.2, 162.7; ESIMS $\mathrm{m} / z$ 412.0 (M $+\mathrm{H})^{+}, 450.1(\mathrm{M}+\mathrm{K})^{+}, 823.2(2 \mathrm{M}+\mathrm{H})^{+}, 845.1(2 \mathrm{M}+\mathrm{Na})^{+}$; $\mathrm{FAB}+\mathrm{m} / z 411\left(\mathrm{M}+\mathrm{e}^{-}\right)^{+}, 434(\mathrm{M}+\mathrm{Na})^{+}$; HRMS calcd for $\mathrm{C}_{18} \mathrm{H}_{25} \mathrm{NO}_{4} \mathrm{SSi} 411.1172$, found 411.1180 .

Methyl 2-(4-Chlorophenyl)-1-(2-(trimethylsilyl)ethylsulfonyl)-2,5-dihydro-1H-pyrrole-3-carboxylate (7k): Thermal Conditions. The crude product was purified by silica gel chromatography $\left(\mathrm{Et}_{2} \mathrm{O} /\right.$ hexane $\left.=3 / 7\right)$ to yield $77 \mathrm{mg}$ $(95 \%)$ of the title compound ${ }^{4 a}$ as a white solid.

Microwave Irradiation. Cyclization of the compound $\mathbf{6 k}$ yielded $1.5 \mathrm{mg}(95 \%)$ of the title compound.

Mp 86.1-87.4 ${ }^{\circ} \mathrm{C}$ dec; IR 2950 (m), 1733 (s), 1346 (s) cm $\mathrm{cm}^{-1}$ ${ }^{1} \mathrm{H}$ NMR $\left(\mathrm{CDCl}_{3}, \mathrm{Me}_{4} \mathrm{Si}\right) \delta-0.11(\mathrm{~s}, 9 \mathrm{H}), 0.55-0.95(\mathrm{~m}, 2 \mathrm{H})$, $2.25-2.60(\mathrm{~m}, 2 \mathrm{H}), 3.66(\mathrm{~s}, 3 \mathrm{H}), 4.40\left(\mathrm{ddd}, 1 \mathrm{H}, J_{2}=17.1 \mathrm{~Hz}\right.$, $\left.J_{3}=6.1 \mathrm{~Hz}, J_{4}=2.0 \mathrm{~Hz}\right), 4.77\left(\mathrm{dt}, 1 \mathrm{H}, J_{2}=17.1 \mathrm{~Hz}, J_{3}=2.6\right.$ $\mathrm{Hz}$ ), $5.83\left(\mathrm{ddd}, 1 \mathrm{H}, J_{4}=6.1 \mathrm{~Hz}, J_{4}=2.6 \mathrm{~Hz}, J_{4}=1.7 \mathrm{~Hz}\right.$ ), $6.96\left(\mathrm{dt}, 1 \mathrm{H}, J_{3}=2.2 \mathrm{~Hz}, J_{4}=1.9 \mathrm{~Hz}\right), 7.35-7.40(\mathrm{~m}, 4 \mathrm{H}) ;{ }^{13} \mathrm{C}$ $\operatorname{NMR}\left(\mathrm{CDCl}_{3}, \mathrm{Me}_{4} \mathrm{Si}\right) \delta-1.8,10.3,50.3,52.4,55.9,68.2,129.35$, 129.6, 134.9, 135.4, 136.8, 138.8, 162.5; ESIMS $\mathrm{m} / \mathrm{z} 402.0(\mathrm{M}$ $+\mathrm{H})^{+} ; \mathrm{FAB}+m / z 402(\mathrm{M}+\mathrm{H})^{+}, 424(\mathrm{M}+\mathrm{Na})^{+}$; HRMS calcd for $\mathrm{C}_{17} \mathrm{H}_{25} \mathrm{ClNO}_{4} \mathrm{SSi}$ 402.0962, found 402.0955.

Methyl 2-(2-Iodophenyl)-1-(2-(trimethylsilyl)ethylsulfonyl)-2,5-dihydro-1H-pyrrole-3-carboxylate ( $7 l)$ : Thermal Conditions. The crude product was purified by silica gel chromatography $\left(\mathrm{Et}_{2} \mathrm{O} /\right.$ hexane $\left.=3 / 7\right)$ to yield $93 \mathrm{mg}(94 \%)$ of the title compound as a white solid.

Mp 134.1-134. ${ }^{\circ} \mathrm{C}$ dec; IR 2948 (m), 1729 (s), 1341 (s) cm ${ }^{-1}$; ${ }^{1} \mathrm{H} \mathrm{NMR}\left(\mathrm{CDCl}_{3}, \mathrm{Me}_{4} \mathrm{Si}\right) \delta-0.11(\mathrm{~s}, 9 \mathrm{H}), 0.65-1.00(\mathrm{~m}, 2 \mathrm{H})$, $2.35-2.70(\mathrm{~m}, 2 \mathrm{H}), 3.66(\mathrm{~s}, 3 \mathrm{H}), 4.50\left(\mathrm{ddd}, 1 \mathrm{H}, J_{2}=17.3 \mathrm{~Hz}\right.$, $\left.J_{3}=6.3 \mathrm{~Hz}, J_{4}=2.0 \mathrm{~Hz}\right), 4.82\left(\mathrm{dt}, 1 \mathrm{H}, J_{2}=17.3 \mathrm{~Hz}, J_{3}=2.6\right.$ $\mathrm{Hz}), 6.21$ (ddd, $1 \mathrm{H}, J_{4}=6.1 \mathrm{~Hz}, J_{4}=2.8 \mathrm{~Hz}, J_{4}=2.0 \mathrm{~Hz}$ ), $6.90-7.10(\mathrm{~m}, 2 \mathrm{H}), 7.20-7.45(\mathrm{~m}, 2 \mathrm{H}), 7.85-7.95(\mathrm{~m}, 1 \mathrm{H}) ;{ }^{13} \mathrm{C}$ $\mathrm{NMR}\left(\mathrm{CDCl}_{3}, \mathrm{Me}_{4} \mathrm{Si}\right) \delta-2.8,9.1,49.0,51.2,55.3,71.3,98.6$, 127.9, 128.6, 129.3, 134.6, 136.0, 139.6, 141.7, 161.3; ESIMS $\mathrm{m} / \mathrm{z} 494.0(\mathrm{M}+\mathrm{H})^{+}, 987.0(2 \mathrm{M}+\mathrm{H})^{+} ; \mathrm{FAB}+\mathrm{m} / z 494(\mathrm{M}+$ $\mathrm{H})^{+}$; HRMS calcd for $\mathrm{C}_{17} \mathrm{H}_{25} \mathrm{INO}_{4} \mathrm{SSi}$ 494.0318, found 494.0307. 
General Procedure for the Synthesis of Pyrroles 8. To a stirred solution ${ }^{2 \mathrm{y}}$ of 2,5 -dihydropyrrole $7(0.10 \mathrm{mmol})$ in 2 $\mathrm{mL}$ of $\mathrm{DMF}$ was added $t$-BuOK $(37 \mathrm{mg}, 0.50 \mathrm{mmol})$. The mixture was stirred at room temperature for $2 \mathrm{~h}$, then $15 \mathrm{~mL}$ of AcOEt was added and the mixture was neutralized with aq $\mathrm{KHSO}_{4}(1 \%)$ and washed with saturated $\mathrm{NaHCO}_{3}$, water, and brine. The organic layer was dried over $\mathrm{MgSO}_{4}$, filtered, and evaporated. Silica gel chromatography $\left(\mathrm{Et}_{2} \mathrm{O} /\right.$ hexane $)$ yielded the pyrrole $\mathbf{8}$.

Methyl 2-Phenyl-1H-pyrrole-3-carboxylate (8a). The crude product was purified by silica gel chromatography $\left(\mathrm{Et}_{2} \mathrm{O} /\right.$ hexane $=2 / 8)$ to yield $16 \mathrm{mg}(80 \%)$ of the title compound ${ }^{4 \mathrm{a}}$ as a white solid.

Mp 90.0-91.4 ${ }^{\circ} \mathrm{C}$ dec; IR 3463 (m), 2943 (m), 1694 (s), 1290 (s) $\mathrm{cm}^{-1} ;{ }^{1} \mathrm{H}$ NMR (acetone- $\left.d_{6}, \mathrm{Me}_{4} \mathrm{Si}\right) \delta 3.57(\mathrm{~s}, 3 \mathrm{H}), 6.54(\mathrm{t}$, $\left.1 \mathrm{H}, J_{3}=2.8 \mathrm{~Hz}\right), 6.78\left(\mathrm{t}, 1 \mathrm{H}, J_{3}=2.8 \mathrm{~Hz}\right), 7.20-7.35(\mathrm{~m}, 3 \mathrm{H})$, $7.50-7.60(\mathrm{~m}, 2 \mathrm{H}), 10.61$ (large s, $1 \mathrm{H}) ;{ }^{13} \mathrm{C}$ NMR (acetone- $d_{6}$, $\left.\mathrm{Me}_{4} \mathrm{Si}\right) \delta 50.8,112.4,112.6,119.0,128.5,128.7,129.9,133.4$, 137.6, 165.6; ESIMS $\mathrm{m} / z 202.1(\mathrm{M}+\mathrm{H})^{+} ; \mathrm{FAB}+\mathrm{m} / z 201(\mathrm{M}$ $\left.-\mathrm{e}^{-}\right)^{+}, 202(\mathrm{M}+\mathrm{H})^{+}$; HRMS calcd for $\mathrm{C}_{12} \mathrm{H}_{11} \mathrm{NO}_{2}$ 201.0790, found 201.0779.

Methyl 2-(3,5-Dimethoxyphenyl)-1H-pyrrole-3-carboxylate (8b). The crude product was purified by silica gel chromatography $\left(\mathrm{Et}_{2} \mathrm{O} /\right.$ hexane $\left.=3 / 7\right)$ to yield $22 \mathrm{mg}(83 \%)$ of the title compound as a white solid.

Mp 120.9-124.0 ${ }^{\circ} \mathrm{C}$ dec; IR 3380 (m), 2930 (m), 1704 (s), $1306(\mathrm{~s}) \mathrm{cm}^{-1} ;{ }^{1} \mathrm{H}$ NMR (acetone- $\left.d_{6}, \mathrm{Me}_{4} \mathrm{Si}\right) \delta 3.63(\mathrm{~s}, 3 \mathrm{H}), 3.74$ $(\mathrm{s}, 6 \mathrm{H}), 6.40\left(\mathrm{t}, 1 \mathrm{H}, J_{3}=2.3 \mathrm{~Hz}\right), 6.57\left(\mathrm{t}, 1 \mathrm{H}, J_{3}=2.8 \mathrm{~Hz}\right)$, $6.80\left(\mathrm{t}, 1 \mathrm{H}, J_{3}=2.8 \mathrm{~Hz}\right), 6.83\left(\mathrm{~d}, 2 \mathrm{H}, J_{3}=2.3 \mathrm{~Hz}\right), 10.65$ (large $\mathrm{s}, 1 \mathrm{H}) ;{ }^{13} \mathrm{C} \mathrm{NMR}$ (acetone- $\left.d_{6}, \mathrm{Me}_{4} \mathrm{Si}\right) \delta 52.7,57.4,102.6,109.6$, 114.4, 114.7, 120.7, 136.7, 139.0, 163.1, 167.4; ESIMS m/z $262.1(\mathrm{M}+\mathrm{H})^{+} ; \mathrm{FAB}+m / z 261\left(\mathrm{M}-\mathrm{e}^{-}\right)^{+}, 262(\mathrm{M}+\mathrm{H})^{+}$; HRMS calcd for $\mathrm{C}_{14} \mathrm{H}_{15} \mathrm{NO}_{4} 261.1001$, found 261.1030.

Methyl 2-(4-(Methoxycarbonyl)phenyl)-1H-pyrrole-3carboxylate $(\mathbf{8 c})$. The crude product was purified by silica gel chromatography $\left(\mathrm{Et}_{2} \mathrm{O} /\right.$ hexane $\left.=3 / 7\right)$ to yield the title compound along with the product of transesterification $\mathbf{8} \mathbf{c}^{\prime}(\mathbf{8 c} /$ $\mathbf{8} \mathbf{c}^{\prime}=1 / 3$ ).

8c: ${ }^{1} \mathrm{H}$ NMR (acetone- $\left.d_{6}, \mathrm{Me}_{4} \mathrm{Si}\right) \delta 3.63(\mathrm{~s}, 3 \mathrm{H}), 3.83(\mathrm{~s}, 3 \mathrm{H})$, 6.55-6.65 (m, 1H), 6.85-6.90 (m, 1H), 7.70-7.80 (m, 2H), 7.90-8.00 (m, 2H), 10.85 (large $\mathrm{s}, 1 \mathrm{H})$.

Methyl 2-(4-(tert-Butyloxycarbonyl)phenyl)-1H-pyrrole3-carboxylate (8c'). ${ }^{1} \mathrm{H}$ NMR (acetone- $\left.d_{6}, \mathrm{Me}_{4} \mathrm{Si}\right) \delta 1.53(\mathrm{~s}$, $9 \mathrm{H}), 3.63(\mathrm{~s}, 3 \mathrm{H}), 6.55-6.65(\mathrm{~m}, 1 \mathrm{H}), 6.85-6.90(\mathrm{~m}, 1 \mathrm{H}), 7.65-$ $7.75(\mathrm{~m}, 2 \mathrm{H}), 7.85-7.95(\mathrm{~m}, 2 \mathrm{H}), 10.85$ (large $\mathrm{s}, 1 \mathrm{H})$.

Methyl 2-(3-Fluorophenyl)-1H-pyrrole-3-carboxylate (8e). The crude product was purified by silica gel chromatography $\left(\mathrm{Et}_{2} \mathrm{O} /\right.$ hexane $\left.=2 / 8\right)$ to yield $18 \mathrm{mg}(81 \%)$ of the title compound as a white solid.

Mp 106.9-108.3 ${ }^{\circ} \mathrm{C}$ dec; IR 3460 (m), 2945 (m), 1719 (s), $1296(\mathrm{~s}) \mathrm{cm}^{-1} ;{ }^{1} \mathrm{H}$ NMR (acetone- $\left.d_{6}, \mathrm{Me}_{4} \mathrm{Si}\right) \delta 3.64(\mathrm{~s}, 3 \mathrm{H}), 6.59$ $\left(\mathrm{t}, 1 \mathrm{H}, J_{3}=2.8 \mathrm{~Hz}\right), 6.85\left(\mathrm{t}, 1 \mathrm{H}, J_{3}=2.8 \mathrm{~Hz}\right), 7.00-7.10(\mathrm{~m}$, $1 \mathrm{H}), 7.30-7.50(\mathrm{~m}, 4 \mathrm{H}), 10.88$ (large $\mathrm{s}, 1 \mathrm{H}) ;{ }^{13} \mathrm{C}$ NMR (acetone$\left.d_{6}, \mathrm{Me}_{4} \mathrm{Si}\right) \delta 51.0,112.9,113.0,115.1\left(\mathrm{~d}, J_{2}=21.1 \mathrm{~Hz}\right), 116.7$ $\left(\mathrm{d}, J_{2}=23.4 \mathrm{~Hz}\right), 119.5,125.6\left(\mathrm{~d}, J_{4}=3.0 \mathrm{~Hz}\right), 130.5\left(\mathrm{~d}, J_{3}=\right.$ $9.1 \mathrm{~Hz}), 135.4\left(\mathrm{~d}, J_{3}=9.1 \mathrm{~Hz}\right), 135.9,163.2\left(\mathrm{~d}, J_{1}=243.0 \mathrm{~Hz}\right)$, 165.5; ESIMS $m / z 220.1(\mathrm{M}+\mathrm{H})^{+}$; FAB $+m / z 219\left(\mathrm{M}-\mathrm{e}^{-}\right)^{+}$, $220(\mathrm{M}+\mathrm{H})^{+}$; HRMS calcd for $\mathrm{C}_{12} \mathrm{H}_{11} \mathrm{FNO}_{2} 220.0774$, found 220.0784 .

Methyl 2-(2-Bromophenyl)-1H-pyrrole-3-carboxylate (8f). The crude product was purified by silica gel chromatography $\left(\mathrm{Et}_{2} \mathrm{O} /\right.$ hexane $\left.=2 / 8\right)$ to yield $23 \mathrm{mg}(81 \%)$ of the title compound as a white solid.

Mp 145.8-147.4 ${ }^{\circ} \mathrm{C}$ dec; IR 3458 (m), 2948 (m), 1693 (s), $1290(\mathrm{~s}) \mathrm{cm}^{-1} ;{ }^{1} \mathrm{H}$ NMR (acetone- $\left.d_{6}, \mathrm{Me}_{4} \mathrm{Si}\right) \delta 3.48(\mathrm{~s}, 3 \mathrm{H}), 6.52$ $\left(\mathrm{t}, 1 \mathrm{H}, J_{3}=2.8 \mathrm{~Hz}\right), 6.81\left(\mathrm{t}, 1 \mathrm{H}, J_{3}=2.8 \mathrm{~Hz}\right), 7.15-7.35(\mathrm{~m}$, $3 \mathrm{H}), 7.55-7.60(\mathrm{~m}, 1 \mathrm{H}), 10.57$ (large s, $1 \mathrm{H}) ;{ }^{13} \mathrm{C}$ NMR (acetone$\left.d_{6}, \mathrm{Me}_{4} \mathrm{Si}\right) \delta 50.7,111.0,114.4,118.9,125.1,127.7,130.7,133.2$, 133.5, 135.5, 135.9, 165.1; ESIMS $m / z 280.2 / 282.1(\mathrm{M}+\mathrm{H})^{+}$;
FAB $+m / z 280 / 282(\mathrm{M}+\mathrm{H})^{+}$; HRMS calcd for $\mathrm{C}_{12} \mathrm{H}_{11} \mathrm{BrNO}_{2}$ 279.9973 , found 279.9960 .

Methyl 2-(Naphthalen-2-yl)-1H-pyrrole-3-carboxylate (8g). The crude product was purified by silica gel chromatography $\left(\mathrm{Et}_{2} \mathrm{O} /\right.$ hexane $\left.=2 / 8\right)$ to yield $17 \mathrm{mg}(66 \%)$ of the title compound as a white solid.

Mp 110.6-112.3 ${ }^{\circ} \mathrm{C}$ dec; IR 3463 (m), 2928 (m), 1694 (s), $1284(\mathrm{~s}) \mathrm{cm}^{-1} ;{ }^{1} \mathrm{H}$ NMR (acetone- $\left.d_{6}, \mathrm{Me}_{4} \mathrm{Si}\right) \delta 3.62(\mathrm{~s}, 3 \mathrm{H}), 6.62$ $\left(\mathrm{t}, 1 \mathrm{H}, J_{3}=2.8 \mathrm{~Hz}\right), 6.87\left(\mathrm{t}, 1 \mathrm{H}, J_{3}=2.8 \mathrm{~Hz}\right), 7.40-7.50(\mathrm{~m}$, $2 \mathrm{H}), 7.70-7.90(\mathrm{~m}, 4 \mathrm{H}), 8.05-8.15(\mathrm{~m}, 1 \mathrm{H}) ; 10.80$ (large s, $1 \mathrm{H})$; ${ }^{13} \mathrm{C}$ NMR (acetone- $d_{6}, \mathrm{Me}_{4} \mathrm{Si}$ ) $\delta 50.9,112.8,119.4,127.1,127.1$, $127.9,128.3,128.4,128.5,129.0,131.0,133.8,134.0,137.5$, 165.7; ESIMS $m / z 252.0(\mathrm{M}+\mathrm{H})^{+}, 503.5(2 \mathrm{M}+\mathrm{H})^{+}$; FAB + $m / z 251\left(\mathrm{M}-\mathrm{e}^{-}\right)^{+}, 252(\mathrm{M}+\mathrm{H})^{+}$; HRMS calcd for $\mathrm{C}_{16} \mathrm{H}_{13} \mathrm{NO}_{2}$ 251.0946, found 251.0958.

Methyl 2-m-Tolyl-1H-pyrrole-3-carboxylate (8h). The crude product was purified by silica gel chromatography $\left(\mathrm{Et}_{2} \mathrm{O} /\right.$ hexane $=2 / 8)$ to yield $15 \mathrm{mg}(72 \%)$ of the title compound as a white solid.

Mp 71.1-72.1 ${ }^{\circ} \mathrm{C}$ dec; IR 3458 (m), 2950 (m), 1693 (s), 1293 (s) $\mathrm{cm}^{-1} ;{ }^{1} \mathrm{H}$ NMR (acetone- $\left.d_{6}, \mathrm{Me}_{4} \mathrm{Si}\right) \delta 2.25(\mathrm{~s}, 3 \mathrm{H}), 3.58(\mathrm{~s}$, $3 \mathrm{H}), 6.53\left(\mathrm{t}, 1 \mathrm{H}, J_{3}=2.8 \mathrm{~Hz}\right), 6.77\left(\mathrm{t}, 1 \mathrm{H}, J_{3}=2.8 \mathrm{~Hz}\right), 7.00-$ $7.25(\mathrm{~m}, 2 \mathrm{H}), 7.30-7.40(\mathrm{~m}, 2 \mathrm{H}), 10.59$ (large s, $1 \mathrm{H}) ;{ }^{13} \mathrm{C}$ NMR (acetone- $\left.d_{6}, \mathrm{Me}_{4} \mathrm{Si}\right) \delta 21.4,50.8,112.4,112.6,118.9,127.0$, 128.6, 129.2, 130.4, 133.3, 137.8, 138.0, 165.6; ESIMS m/z $216.2(\mathrm{M}+\mathrm{H})^{+} ; \mathrm{FAB}+m / z 215\left(\mathrm{M}-\mathrm{e}^{-}\right)^{+}, 216(\mathrm{M}+\mathrm{H})^{+}$; HRMS calcd for $\mathrm{C}_{13} \mathrm{H}_{13} \mathrm{NO}_{2} 215.0946$, found 215.0951.

Methyl 2-(3,5-Dimethylphenyl)-1H-pyrrole-3-carboxylate (8i). The crude product was purified by silica gel chromatography $\left(\mathrm{Et}_{2} \mathrm{O} /\right.$ hexane $\left.=2 / 8\right)$ to yield $18 \mathrm{mg}(78 \%)$ of the title compound as a white solid.

Mp 124.8-126.2 ${ }^{\circ} \mathrm{C}$ dec; IR 3463 (m), 2943 (m), 1693 (s), $1303(\mathrm{~s}) \mathrm{cm}^{-1} ;{ }^{1} \mathrm{H}$ NMR (acetone- $\left.d_{6}, \mathrm{Me}_{4} \mathrm{Si}\right) \delta 2.17(\mathrm{~s}, 6 \mathrm{H}), 3.54$ $(\mathrm{s}, 3 \mathrm{H}), 6.48\left(\mathrm{t}, 1 \mathrm{H}, J_{3}=2.8 \mathrm{~Hz}\right), 6.71\left(\mathrm{t}, 1 \mathrm{H}, J_{3}=2.8 \mathrm{~Hz}\right)$, $6.80-6.90(\mathrm{~m}, 1 \mathrm{H}), 7.10-7.20(\mathrm{~m}, 2 \mathrm{H}), 10.49($ large $\mathrm{s}, 1 \mathrm{H}) ;{ }^{13} \mathrm{C}$ NMR (acetone- $\left.d_{6}, \mathrm{Me}_{4} \mathrm{Si}\right) \delta 18.9,48.3,109.9,110.2,116.3$, $125.3,127.6,130.9,135.5,163.2 ;$ ESIMS $m / z 230.2(\mathrm{M}+\mathrm{H})^{+}$; $\mathrm{FAB}+m / z 229\left(\mathrm{M}-\mathrm{e}^{-}\right)^{+}, 230(\mathrm{M}+\mathrm{H})^{+}$; HRMS calcd for $\mathrm{C}_{14} \mathrm{H}_{15} \mathrm{NO}_{2} 229.1103$, found 229.1096 .

Methyl 2-(2,3-Methylenedioxyphenyl)-1H-pyrrole-3carboxylate $(\mathbf{8 j})$. The crude product was purified by silica gel chromatography $\left(\mathrm{Et}_{2} \mathrm{O} /\right.$ hexane $\left.=3 / 7\right)$ to yield $22 \mathrm{mg}(88 \%)$ of the title compound as a white solid.

Mp 101.9-104.7 ${ }^{\circ} \mathrm{C}$ dec; IR 3455 (m), 2968 (m), 1730 (s), $1295(\mathrm{~s}) \mathrm{cm}^{-1} ;{ }^{1} \mathrm{H}$ NMR (acetone- $\left.d_{6}, \mathrm{Me}_{4} \mathrm{Si}\right) \delta 3.62(\mathrm{~s}, 3 \mathrm{H}), 5.93$ $(\mathrm{s}, 2 \mathrm{H}), 6.57\left(\mathrm{t}, 1 \mathrm{H}, J_{3}=2.8 \mathrm{~Hz}\right), 6.75-6.85(\mathrm{~m}, 2 \mathrm{H}), 6.85(\mathrm{t}$, $\left.1 \mathrm{H}, J_{3}=2.8 \mathrm{~Hz}\right), 7.10-7.20(\mathrm{~m}, 1 \mathrm{H}), 10.61($ large s, $1 \mathrm{H}) ;{ }^{13} \mathrm{C}$ NMR (acetone- $\left.d_{6}, \mathrm{Me}_{4} \mathrm{Si}\right) \delta 50.9,101.7,108.6,112.2,114.1$, $115.8,119.3,121.9,123.8,131.3,145.8,148.3,165.5$; ESIMS $m / z 246.0(\mathrm{M}+\mathrm{H})^{+} ; \mathrm{FAB}+m / z 245\left(\mathrm{M}-\mathrm{e}^{-}\right)^{+}, 246(\mathrm{M}+\mathrm{H})^{+}$; HRMS calcd for $\mathrm{C}_{13} \mathrm{H}_{11} \mathrm{NO}_{4} 245.0688$, found 245.0678 .

Methyl 2-(4-Chlorophenyl)-1H-pyrrole-3-carboxylate (8k). The crude product was purified by silica gel chromatography $\left(\mathrm{Et}_{2} \mathrm{O} /\right.$ hexane $\left.=2 / 8\right)$ to yield $19 \mathrm{mg}(81 \%)$ of the title compound $^{4 \mathrm{a}}$ as a white solid.

Mp 113.3-114.5 ${ }^{\circ} \mathrm{C}$ dec; IR 3380 (m), 2948 (m), 1695 (s), $1286(\mathrm{~s}) \mathrm{cm}^{-1} ;{ }^{1} \mathrm{H}$ NMR (acetone- $\left.d_{6}, \mathrm{Me}_{4} \mathrm{Si}\right) \delta 3.62(\mathrm{~s}, 3 \mathrm{H}), 6.58$ $\left(\mathrm{t}, 1 \mathrm{H}, J_{3}=2.8 \mathrm{~Hz}\right), 6.84\left(\mathrm{t}, 1 \mathrm{H}, J_{3}=2.8 \mathrm{~Hz}\right), 7.30-7.40(\mathrm{~m}$, $2 \mathrm{H}$ ), 7.55-7.65 (m, 2H), 10.75 (large s, $1 \mathrm{H}) ;{ }^{13} \mathrm{C}$ NMR (acetone$\left.d_{6}, \mathrm{Me}_{4} \mathrm{Si}\right) \delta 50.9,112.8,112.9,119.4,128.7,131.5,132.1,133.9$, 136.1, 165.5; ESIMS $m / z 236.3(\mathrm{M}+\mathrm{H})^{+}$; FAB $+m / z 235(\mathrm{M}$ $\left.-\mathrm{e}^{-}\right)^{+}, 236(\mathrm{M}+\mathrm{H})^{+} ; \mathrm{HRMS}$ calcd for $\mathrm{C}_{12} \mathrm{H}_{10} \mathrm{ClNO}_{2} 235.0400$, found 235.0383 .

Methyl 2-(2-Iodophenyl)-1H-pyrrole-3-carboxylate (8l). The crude product was purified by silica gel chromatography $\left(\mathrm{Et}_{2} \mathrm{O} /\right.$ hexane $\left.=2 / 8\right)$ to yield $27 \mathrm{mg}(81 \%)$ of the title compound as a white solid.

Mp 140.4-144.8 ${ }^{\circ} \mathrm{C}$ dec; IR 3465 (m), 2950 (m), 1711 (s), $1275(\mathrm{~s}) \mathrm{cm}^{-1} ;{ }^{1} \mathrm{H}$ NMR (acetone- $\left.d_{6}, \mathrm{Me}_{4} \mathrm{Si}\right) \delta 3.47(\mathrm{~s}, 3 \mathrm{H}), 6.51$ $\left(\mathrm{t}, 1 \mathrm{H}, J_{3}=2.8 \mathrm{~Hz}\right), 6.79\left(\mathrm{t}, 1 \mathrm{H}, J_{3}=2.8 \mathrm{~Hz}\right), 7.05(\mathrm{ddd}, 1 \mathrm{H}$, $\left.J_{3}=7.9 \mathrm{~Hz}, J_{3}=7.3 \mathrm{~Hz}, J_{4}=1.9 \mathrm{~Hz}\right), 7.26\left(\mathrm{dd}, 1 \mathrm{H}, J_{3}=7.6\right.$ $\left.\mathrm{Hz}, J_{4}=1.9 \mathrm{~Hz}\right), 7.34\left(\mathrm{ddd}, 1 \mathrm{H}, J_{3}=7.6 \mathrm{~Hz}, J_{3}=7.3 \mathrm{~Hz}, J_{4}\right.$ 
$=1.2 \mathrm{~Hz}$ ), $7.83\left(\mathrm{dd}, 1 \mathrm{H}, J_{3}=7.9 \mathrm{~Hz}, J_{4}=1.2 \mathrm{~Hz}\right), 10.50$ (large $\mathrm{s}, 1 \mathrm{H}$ ); ${ }^{13} \mathrm{C}$ NMR (acetone- $d_{6}, \mathrm{Me}_{4} \mathrm{Si}$ ) $\delta 50.7,101.1,110.9,114.1$, 118.7, 128.4, 130.63, 132.4, 139.1, 139.5, 139.9, 165.0; ESIMS $m / z 328.1(\mathrm{M}+\mathrm{H})^{+} ; \mathrm{FAB}+\mathrm{m} / z 328(\mathrm{M}+\mathrm{H})^{+} ;$HRMS calcd for $\mathrm{C}_{12} \mathrm{H}_{11} \mathrm{INO}_{2} 327.9835$, found 327.9863 .

Acknowledgment. We thank the CNRS and the MENRT for financial support. The technical support from Biotage (Personal Chemistry) and CEM for the microwave-activated experiments is acknowledged. 